International Journal of Modern Physics B, (C) World Scientific Publishing Company

\title{
THE BROWNIAN VACANCY DRIVEN WALK
}

\author{
ZOLTÁN TOROCZKAI \\ Center for Stochastic Processes in Science and Engineering, and \\ Department of Physics, Virginia Polytechnic Institute and State University, \\ Blacksburg, Virginia 24061-0435, USA
}

Received 27 May 1997

\begin{abstract}
We investigate the lattice walk performed by a tagged member of an infinite 'sea' of particles filling a d-dimensional lattice, in the presence of a Brownian vacancy. Particleparticle exchange is forbidden; the only interaction between them being hard core exclusion. The tagged particle, differing from the others only by its tag, moves only when it exchanges places with the hole. In this sense, it is a lattice walk "driven" by the Brownian vacancy. The probability distributions for its displacement and for the number of steps taken, after $n$-steps of the vacancy, are derived. Surprisingly, none of them is a Gaussian! It is shown that the only nontrivial dimension where the walk is recurrent is $d=2$.
\end{abstract}

\section{Introduction}

In solids, long-range diffusion of atoms mediated by vacancies (the so-called

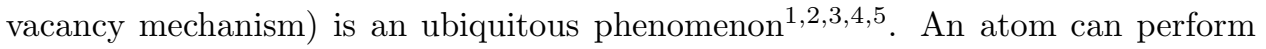
a displacement only if there is a neighbouring vacancy. For low enough vacancy densities the motion performed by each of these vacancies can be considered as a simple (Brownian) random walk on the crystalline lattice, independent of the other walkers. Let us tag one of the atoms. Due to encounters with the vacancies, this atom performs some kind of random walk. One question naturally arises: what are the properties of this walk? Specifically, we may ask: What is the probability distribution to find this atom at a certain site? How long does it take, on the average, to get there, etc? One central result of this study is that the probability distributions are not Gaussians, being very differrent from the walk performed by the vacancy. Since this walk is "driven" by a Brownian hole, we introduced a name for it: "the Brownian vacancy driven walk".

The precise formulation of this problem is stated in Section 2 together with the derivation of the complete probability distribution characterizing the couple tagvacancy. In Section 3 we cover some important relations from the theory of lattice walks and walks with taboo sites, so that in Section 4, we can explicitely present the probability distributions for the displacement and for the number of steps the tagged particle made. It is also shown that the only non-trivial dimension where the walk is recurrent is $d=2$.

Before we present our analysis, we remark that, after completion of our work, 
we were informed (Krapivsky) of the study by Brummelhuis and Hilhorst 17 . These authors considered the same problem, but only in two dimensions. Our approach differs from theirs, in that we considered systems in arbitrary dimensions: $d$. By leaving $d$ as a parameter in the theory, we see the uniqueness of $d=2$ systems within this wider context. Further, we have analyzed several other quantities absent in Ref. 17. See, for example, Section 4.1. Of course, where we do have overlap, the results of 17 are reproduced exactly.

\section{Formulation of the problem and construction of the probability dis- tribution}

Consider a $d$-dimensional hypercubic, infinite lattice. Assume, that every lattice site is occupied by a single "white" particle (or ball) except for two sites: one of

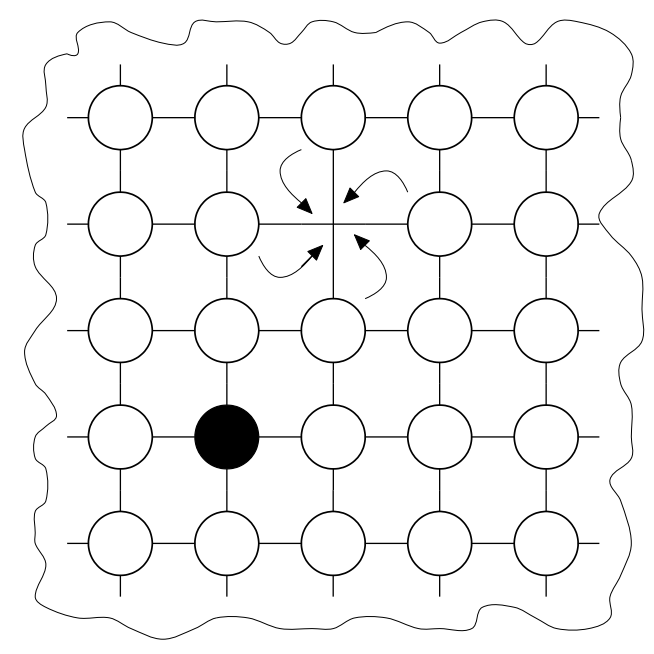

Fig. 1. The infinite sea of particles with one tagged (the solid circle) particle, and one empty site.

them is empty (called "hole" or vacancy) and the other is occupied by a "black" colored ball, or particle, see Fig. 1.

The system evolves according to the following rules:

1) There is only an excluded volume constraint on the balls; no other interactions are present.

2) The balls can take one step (equal to one lattice constant) jumps and only onto the empty site.

3) The probability that the empty site will be occupied by one of its nearest neighbours is the same for every neighbour: $p=1 / 2 d$.

4) The black ball is distinguished from the others only by its color. Otherwise it obeys exactly the same rules 1)-3) as the others.

It is obvious that the hole performs random walk of Pólya type 6. 6 . 
rule 2), the black ball performs a "passive" walk, moving only as a result of being "kicked" by the vacancy. One might as well say that it is driven by the Brownian vacancy. Our main concern is the motion of the colored ball. More precisely various properties of the walk performed by the black ball will be derived rigorously.

In the following we write down the full probability distribution for the pair: vacancy and tagged particle. We have also found an alternative, and mathematically more rigurous derivation 9 than the one presented below, however the version presented here is much shorter, and perhaps more intuitive, too.

Without loss of generality let us assume that, initially, the black ball is in the origin, and the hole is at $\mathbf{r}_{\mathbf{0}}\left(\mathbf{r}_{\mathbf{0}} \neq \mathbf{0}\right)\left(\mathbf{r}_{\mathbf{0}} \in Z^{d}\right)$. After $n$ steps by the hole, the black ball is to be found at site $\boldsymbol{\rho}$ and the hole, at site $\boldsymbol{\rho}+\mathbf{r}$, see Fig. 2 . $\left(\boldsymbol{\rho}, \mathbf{r} \in Z^{d}\right.$, $\mathbf{r} \neq \mathbf{0})$. For later convenience, let us denote: $\mathbf{R} \equiv \boldsymbol{\rho}+\mathbf{r}$.

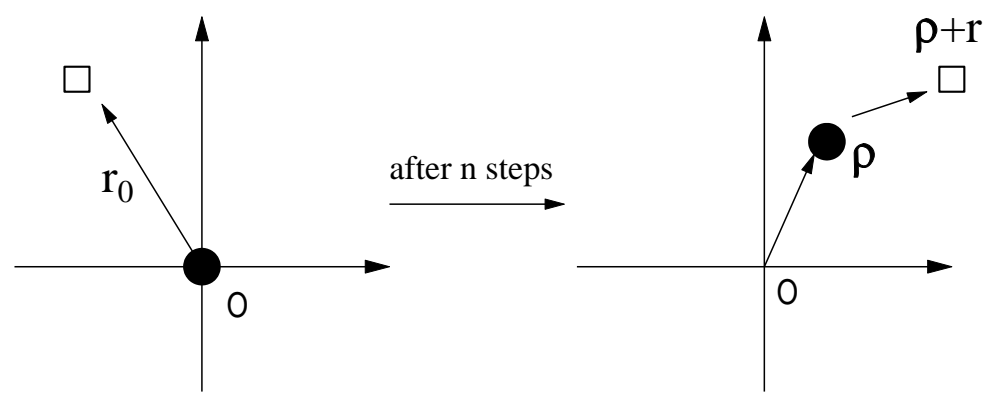

Fig. 2. Initially the tagged particle (the solid circle) is in the origin, and the vacancy is at site $\mathbf{r}_{\mathbf{0}}$ (the empty square). After $n$ steps the tagged particle is at site $\rho$ and the random walker at site $\mathbf{R}=\rho+\mathbf{r}$

Let us introduce the following notations:

- $P_{n}\left(\mathbf{s} \mid \mathbf{s}_{\mathbf{0}}\right)$ is the probability that the vacancy is found at site $\mathbf{s}$ after $n$ steps, given it started at $\mathbf{s}_{\mathbf{0}}, n \geq 1$, and $\mathbf{s}, \mathbf{s}_{\mathbf{0}} \in Z^{d}$. For $n=0$ by convention, $P_{0}\left(\mathbf{s} \mid \mathbf{s}_{\mathbf{0}}\right)=\delta_{\mathbf{s}, \mathbf{s}} \mathbf{0}$

- $P_{n}^{\dagger}\left(\mathbf{s} \mid \mathbf{s}_{\mathbf{0}}\right)$ is the probability that the vacancy is found at site $\mathbf{s}\left(\mathbf{s}, \mathbf{s}_{\mathbf{0}} \neq \mathbf{0}\right)$ after $n$ steps in the presence of an irreversible trap at site $\mathbf{0}$, given the walk commenced at site $\mathbf{s}_{\mathbf{0}}$. In other words, $P_{n}^{\dagger}\left(\mathbf{s}_{\mathbf{s}} \mid \mathbf{s}_{\mathbf{0}}\right)$ is just the probability to get to $\mathbf{s}$ from $\mathbf{s}_{\mathbf{0}}$ in $n$ steps without having visited the origin. In the literature, the $P_{n}^{\dagger}\left(\mathbf{s} \mid \mathbf{s}_{\mathbf{0}}\right)$ 's are called 'taboo' probabilities, see Ref. 6. Consistently, $P_{0}^{\dagger}\left(\mathbf{s}^{\prime} \mid \mathbf{s}\right)=\delta_{\mathbf{s}^{\prime}, \mathbf{s}}$.

- $\pi_{n}^{k}\left(\boldsymbol{\rho}, \mathbf{r} \mid \mathbf{0}, \mathbf{r}_{\mathbf{0}}\right)$ is the probability that the black ball was driven $k$ steps from $\mathbf{0}$ to $\boldsymbol{\rho}$, during which the random walker made $n$ steps from $\mathbf{r}_{\mathbf{0}}$ to $\mathbf{r}+\boldsymbol{\rho}$.

Furthermore, $\sum_{\mathbf{S}}^{\left(\mathbf{s}_{\mathbf{0}}\right)}$ will mean summing over all the nearest neighbours of site $\mathbf{s}_{\mathbf{0}}$, and in $\sum_{\mathbf{S}}$ the summation is taken over all the lattice sites. 
Let us consider the referrence frame connected to the tagged particle. In this frame the walk performed by the vacancy away from the origin is just the regular Pólya walk, but it can never visit the origin. Instead it "jumps over" the origin, by moving two lattice constants. This jump corresponds to the simple exchange of the vacancy and tagged particle in the original frame. With this observation,

$$
\begin{array}{r}
\pi_{n}^{k}\left(\boldsymbol{\rho}, \mathbf{r} \mid \mathbf{0}, \mathbf{r}_{\mathbf{0}}\right)=p^{k} \sum_{\nu_{1}}^{(\mathbf{0})} \sum_{\nu_{2}}^{(\mathbf{0})} \ldots \sum_{\nu_{k}}^{(\mathbf{0})} \sum_{n_{0}=1}^{\infty} \sum_{n_{1}=1}^{\infty} \ldots \sum_{n_{k}=0}^{\infty} \delta_{n, \sum_{i=1}^{k}{ }_{n_{i}} \delta_{\rho, \sum_{i=1}^{k} \nu_{i}}} \\
P_{n_{k}}^{\dagger}\left(\mathbf{r} \mid-\boldsymbol{\nu}_{k}\right) P_{n_{k-1}-1}^{\dagger}\left(\boldsymbol{\nu}_{k} \mid-\boldsymbol{\nu}_{k-1}\right) \ldots P_{n_{1}-1}^{\dagger}\left(\boldsymbol{\nu}_{2} \mid-\boldsymbol{\nu}_{1}\right) P_{n_{0}-1}^{\dagger}\left(\boldsymbol{\nu}_{1} \mid \mathbf{r}_{\mathbf{0}}\right), \\
k \geq 1, \quad \mathbf{r}_{\mathbf{0}}, \mathbf{r} \neq 0 .
\end{array}
$$

We had to sum over all the partitions of the time $n$ into $k$ subsets, as well a sum over all the possible directions (in number of $2 d$ ) from which the black ball can be "hit". Obviously if $\boldsymbol{\nu}_{i}$ is a nearest neighbour of the origin, then $-\boldsymbol{\nu}_{i}$ is another nearest neighbour on the other side of the origin, straight accross.

The $k=0$ case has to be treated separately:

$$
\pi_{n}^{0}\left(\boldsymbol{\rho}, \mathbf{r} \mid \mathbf{0}, \mathbf{r}_{\mathbf{0}}\right)=\delta_{\rho, \mathbf{0}} P_{n}^{\dagger}\left(\mathbf{r} \mid \mathbf{r}_{\mathbf{0}}\right) .
$$

By analyzing (1), we infer: $\pi_{n}^{k}\left(\boldsymbol{\rho}, \mathbf{r} \mid \mathbf{0}, \mathbf{r}_{\mathbf{0}}\right)=0$, if $k>n$, as it should be, since the black ball cannot be hit more times than the number of steps taken by the random walker.

\section{Generating functions}

Usually, it is more convenient to work with generating functions, instead of the distributions, themselves. In general, if the generating function for the distribution $G_{n}$, i.e., $G(\xi)=\sum_{n=0}^{\infty} \xi^{n} G_{n}$ is known, the $G_{n}$ 's are retrieved by the complex integral along the contour $\mathcal{C}$ encircling the origin (counterclockwise)::

$$
G_{n}=\frac{1}{2 \pi \mathrm{i}} \oint_{\mathcal{C}} \frac{d \xi}{\xi^{n+1}} G(\xi) .
$$

One can also quote the following relations, to be used extensively in our treatment:

$$
\sum_{j=0}^{n} G_{j}=\frac{1}{2 \pi \mathrm{i}} \oint_{\mathcal{C}} \frac{d \xi}{\xi^{n+1}} \frac{1}{1-\xi} G(\xi), \text { and } \sum_{j=0}^{\infty} G_{j}=G(1) .
$$

In the case of the Pólya walk, the generating function is expressed as 6.11:

$$
P\left(\mathbf{s} \mid \mathbf{s}_{\mathbf{0}} ; \xi\right)=\int_{-\pi}^{\pi} \frac{d^{d} l}{(2 \pi)^{d}} \frac{e^{\mathrm{il}\left(\mathbf{s}-\mathbf{s}_{\mathbf{0}}\right)}}{1-\xi \omega(\mathbf{l})}
$$

where the integral is taken over the first Brillouin zone $[-\pi, \pi]^{d}$, and $\omega$ is the structure function of the walk $\omega(\mathbf{l})=\frac{1}{d} \sum_{j=1}^{d} \cos \left(l_{j}\right),-\pi \leq l_{j} \leq \pi\left(\mathbf{l}=\sum_{j=1}^{d} l_{j} \mathbf{e}_{\mathbf{j}}\right.$ with $\left\{\mathbf{e}_{\mathbf{j}}\right\}$ as unit vectors in $\mathbb{R}^{d}$. 
For the simplicity of writing, let us introduce the following notations:

$$
\begin{aligned}
& t=t(\xi) \equiv P(\mathbf{0} \mid \mathbf{0} ; \xi), \\
& u=u(\xi) \equiv P(\boldsymbol{\alpha} \mid \mathbf{0} ; \xi), \\
& h=h(\xi) \equiv P(\boldsymbol{\alpha} \mid-\boldsymbol{\alpha} ; \xi), \\
& b=b(\xi) \equiv P(\boldsymbol{\beta} \mid-\boldsymbol{\alpha} ; \xi), \quad \boldsymbol{\beta} \neq \pm \boldsymbol{\alpha}, \quad \text { (only for } d \geq 2)
\end{aligned}
$$

where $\boldsymbol{\alpha}$ and $\boldsymbol{\beta}$ are nearest neighbours of the origin $(|\boldsymbol{\alpha}|=1,|\boldsymbol{\beta}|=1) . t$ is the generating function for the return probabilities, $u$ is for the probabilities of return to a nearest neighbour, etc.., see Fig. 3 .

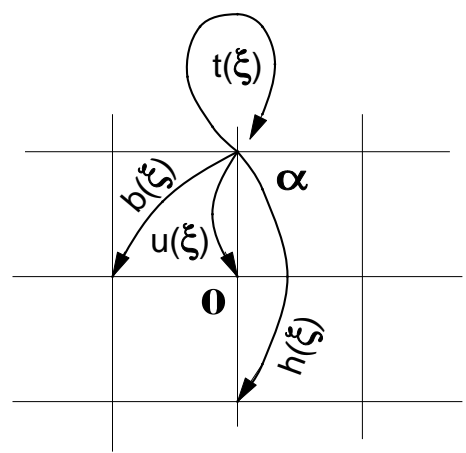

Fig. 3. The generating functions $t, h, b$ and $u$.

The connection between the taboo and Pólya walk probabilities is most simply given in terms of generating functions 6 :

$$
P^{\dagger}\left(\mathbf{s} \mid \mathbf{s}_{\mathbf{0}} ; \xi\right)=P\left(\mathbf{s} \mid \mathbf{s}_{\mathbf{0}} ; \xi\right)-\frac{1}{t} P(\mathbf{s} \mid \mathbf{0} ; \xi) P\left(\mathbf{0} \mid \mathbf{s}_{\mathbf{0}} ; \xi\right), \quad \mathbf{s}, \mathbf{s}_{\mathbf{0}} \neq \mathbf{0}
$$

Next, consider the identitie 12 :

$$
\begin{aligned}
& \sum_{\mathbf{S}} e^{\mathrm{i} \mathbf{l} \mathbf{s}}=(2 \pi)^{d} \delta(\mathbf{l}), \quad s \in Z^{d}, \quad \mathbf{l} \in[-\pi, \pi]^{d}, \\
& \sum_{\beta}^{(\mathbf{0})} e^{\mathrm{i} \mathbf{l} \beta}=\frac{1}{p} \omega(\mathbf{l}), \quad \mathbf{l} \in[-\pi, \pi]^{d}, \\
& \int_{-\pi}^{\pi} \frac{d^{d} l}{(2 \pi)^{d}} e^{\mathrm{i} \mathbf{l} \mathbf{s}}=\delta_{\mathbf{s}, \mathbf{0}}, \quad \mathbf{s} \in Z^{d}, \quad \mathbf{l} \in[-\pi, \pi]^{d} .
\end{aligned}
$$

In view of the above, the following useful relations valid for any $\mathbf{l} \in[-\pi, \pi]^{d}$ and $\mathbf{s}_{\mathbf{1}}, \mathbf{s}_{\mathbf{0}} \in Z^{d}$, are easily proved :

$$
\sum_{\mathbf{s}} e^{\mathrm{il} \mathbf{l}} P\left(\mathbf{s} \mid \mathbf{s}_{\mathbf{0}} ; \xi\right)=\frac{e^{\mathrm{i} \mathbf{l} \mathbf{s}} \mathbf{0}}{1-\xi \omega(\mathbf{l})},
$$


Z. Toroczkai

$$
\sum_{\alpha}^{\left(\mathbf{s}_{\mathbf{1}}\right)} e^{\mathrm{i} \mathbf{l} \alpha} P\left(\boldsymbol{\alpha} \mid \mathbf{s}_{\mathbf{0}} ; \xi\right)=\frac{e^{\mathrm{i} \mathbf{l} \mathbf{s}_{\mathbf{1}}}}{p \xi} \int_{-\pi}^{\pi} \frac{d^{d} \chi}{(2 \pi)^{d}} \frac{\xi \omega(\boldsymbol{\chi}-\mathbf{l})}{1-\xi \omega(\boldsymbol{\chi})} e^{\mathrm{i} \chi\left(\mathbf{s}_{\mathbf{1}}{ }^{\left.-\mathbf{s}_{\mathbf{0}}\right)}\right.}
$$

By inserting $\mathbf{l}=\mathbf{0}$ in (15) and using (5) and (13), is obtained:

$$
\sum_{\alpha}^{\left(\mathbf{s}_{\mathbf{1}}\right)} P\left(\boldsymbol{\alpha} \mid \mathbf{s}_{\mathbf{0}} ; \xi\right)=\frac{1}{p \xi}\left[P\left(\mathbf{s}_{\mathbf{1}} \mid \mathbf{s}_{\mathbf{0}} ; \xi\right)-\delta_{\left.\mathbf{s}_{\mathbf{1}}, \mathbf{s}_{\mathbf{0}}\right]}\right]
$$

A further consequence is with $\mathbf{s}_{\mathbf{1}}=\mathbf{0}$ ( $\boldsymbol{\alpha}$ is a nearest neighbour of the origin) and $\mathbf{s}_{\mathbf{0}}=\mathbf{0}$ in (16). Then, in virtue of notations (6-7) the left hand side becomes $2 d u$, whereas the right hand side $(t-1) / p \xi$. Thus:

$$
u=\frac{t-1}{\xi} .
$$

Consider now $\mathbf{s}_{\mathbf{1}}=\mathbf{0}$ and $\mathbf{s}_{\mathbf{0}} \equiv \boldsymbol{\beta}$ be a nearest neighbour of the origin. Then from (16):

$$
\sum_{\alpha}^{(\mathbf{0})} P(\boldsymbol{\alpha} \mid \boldsymbol{\beta} ; \xi)=t+2(d-1) b+h=\frac{u}{p \xi} .
$$

Let us now assume the notations: $\sum_{\mathbf{S}}^{\prime}$ will mean summing over all the sites except for $\mathbf{s}=\mathbf{0} ; \sum_{\mathbf{s}}^{\left(\mathbf{s}_{\mathbf{1}}\right)}$, will mean summing over all the nearest neighbour sites of $\mathbf{s}_{\mathbf{1}}$ except for $\mathbf{s}=\mathbf{0}$ (if that is the case).

Similarly to Eqs. (14 15 ) one can write : for any $\mathbf{l} \in[-\pi, \pi]^{d}$ and $\mathbf{s}_{\mathbf{1}}, \mathbf{s}_{\mathbf{0}} \in Z^{d}$, $\mathrm{s}_{\mathbf{0}} \neq \mathbf{0}$ :

$$
\begin{gathered}
\sum_{\mathbf{s}}^{\prime} e^{\mathrm{i} \mathbf{l} \mathbf{s}} P^{\dagger}\left(\mathbf{s} \mid \mathbf{s}_{\mathbf{0}} ; \xi\right)=\frac{e^{\mathrm{il} \mathbf{s} \mathbf{0}}}{1-\xi \omega(\mathbf{l})}\left[1-\frac{e^{\mathrm{i} \mathbf{l} \mathbf{0}}}{t} P\left(\mathbf{0} \mid \mathbf{s}_{\mathbf{0}} ; \xi\right)\right] \\
\sum_{\alpha}^{\left(\mathbf{s}_{\mathbf{1}}\right)} e^{\mathrm{i} \mathbf{l} \alpha} P^{\dagger}\left(\boldsymbol{\alpha} \mid \mathbf{s}_{\mathbf{0}} ; \xi\right)=\frac{e^{\mathrm{i} \mathbf{l} \mathbf{s}_{\mathbf{1}}}}{p \xi} \int_{-\pi}^{\pi} \frac{d^{d} \chi}{(2 \pi)^{d}} \frac{\xi \omega(\boldsymbol{\chi}-\mathbf{l})}{1-\xi \omega(\boldsymbol{\chi})} e^{\mathrm{i} \chi\left(\mathbf{s}_{\mathbf{1}}-\mathbf{s}_{\mathbf{0}}\right)}\left[1-\frac{e^{\mathrm{i} \chi \mathbf{s}_{\mathbf{0}}}}{t} P\left(\mathbf{0} \mid \mathbf{s}_{\mathbf{0}} ; \xi\right)\right] .
\end{gathered}
$$

(19) and (20) can easily be proved using (10) and (14),(15).

A list of useful particular cases:

$$
\begin{gathered}
\sum_{\mathbf{S}}^{\prime} e^{\mathrm{i} \mathbf{l} \mathbf{s}} P^{\dagger}(\mathbf{s} \mid \boldsymbol{\alpha} ; \xi)=\frac{e^{-\mathrm{i} \mathbf{l} \alpha}}{1-\xi \omega(\mathbf{l})}\left(1-\frac{u}{t} e^{\mathrm{il} \mathbf{l}}\right) \\
\sum_{\mathbf{S}}^{\prime} P^{\dagger}\left(\mathbf{s} \mid \mathbf{s}_{\mathbf{0}} ; \xi\right)=\frac{1}{1-\xi}\left[1-\frac{P\left(\mathbf{0} \mid \mathbf{s}_{\mathbf{0}} ; \xi\right)}{t}\right] \\
\sum_{\mathbf{S}}^{\prime} P^{\dagger}(\mathbf{s} \mid \boldsymbol{\alpha} ; \xi)=\frac{1}{1-\xi}\left(1-\frac{u}{t}\right)
\end{gathered}
$$




$$
\sum_{\beta}^{(\mathbf{0})} P^{\dagger}\left(\boldsymbol{\beta} \mid \mathbf{s}_{\mathbf{0}} ; \xi\right)=\frac{1}{p \xi} \frac{P\left(\mathbf{0} \mid \mathbf{s}_{\mathbf{0}} ; \xi\right)}{t}
$$

and

$$
\sum_{\beta}^{(\mathbf{0})} P^{\dagger}(\boldsymbol{\beta} \mid \boldsymbol{\alpha} ; \xi)=\frac{1}{p \xi} \frac{u}{t},
$$

with $\boldsymbol{\alpha}$ a nearest neighbour of the origin and $\mathbf{s}_{\mathbf{0}} \neq \mathbf{0}$.

Let us now return to our original problem, and trade the discrete time variable $n$ for the continuous, complex variable $\xi$, via:

$$
\Pi^{k}\left(\boldsymbol{\rho}, \mathbf{r} \mid \mathbf{0}, \mathbf{r}_{\mathbf{0}} ; \xi\right)=\sum_{n=0}^{\infty} \xi^{n} \pi_{n}^{k}\left(\boldsymbol{\rho}, \mathbf{r} \mid \mathbf{0}, \mathbf{r}_{\mathbf{0}}\right)
$$

From (11) and (2) and using the generating function for the taboo probabilities one finds:

$k \geq 1$ :

$$
\begin{array}{r}
\Pi^{k}\left(\boldsymbol{\rho}, \mathbf{r} \mid \mathbf{0}, \mathbf{r}_{\mathbf{0}} ; \xi\right)=(p \xi)^{k} \sum_{\nu_{1}}^{(\mathbf{0})} \sum_{\nu_{2}}^{(\mathbf{0})} \ldots \sum_{\nu_{k}}^{(\mathbf{0})} \delta \sum_{\rho, \sum_{i=1}^{k} \nu_{i}} P^{\dagger}\left(\mathbf{r} \mid-\boldsymbol{\nu}_{k} ; \xi\right) P^{\dagger}\left(\boldsymbol{\nu}_{k} \mid-\boldsymbol{\nu}_{k-1} ; \xi\right) \ldots \\
\ldots P^{\dagger}\left(\boldsymbol{\nu}_{2} \mid-\boldsymbol{\nu}_{1} ; \xi\right) P^{\dagger}\left(\boldsymbol{\nu}_{1} \mid \mathbf{r}_{\mathbf{0}} ; \xi\right),
\end{array}
$$

$k=0$ :

$$
\Pi^{0}\left(\boldsymbol{\rho}, \mathbf{r} \mid \mathbf{0}, \mathbf{r}_{\mathbf{0}} ; \xi\right)=\delta_{\rho, 0} P^{\dagger}\left(\mathbf{r} \mid \mathbf{r}_{\mathbf{0}} ; \xi\right) .
$$

In Appendix A it is shown that the distribution $\pi_{n}^{k}\left(\boldsymbol{\rho}, \mathbf{r} \mid \mathbf{0}, \mathbf{r}_{\mathbf{0}}\right)$ is normalized. (This also serves as a check for the correctness of (11) and (2).) There we first show

$$
\sum_{k=0}^{\infty} \sum_{\rho} \sum_{\mathbf{r}}^{\prime} \delta_{\mathbf{R}, \rho+\mathbf{r}} \pi_{n}^{k}\left(\boldsymbol{\rho}, \mathbf{r} \mid \mathbf{0}, \mathbf{r}_{\mathbf{0}}\right)=P_{n}\left(\mathbf{R} \mid \mathbf{r}_{\mathbf{0}}\right) .
$$

so that normalization immediately follows $\left(\sum_{\mathbf{R}} P_{n}\left(\mathbf{R} \mid \mathbf{r}_{\mathbf{0}}\right)=1\right)$.

In many cases, the integral in (3) cannot be carried out explicitly. Only asymptotic behavior can be given in some limit. Of course, the limit of most interest here is $n \rightarrow \infty$. For this purpose, we quote an extremely useful theorem, the Discrete Tauberian Theorem 6. 0 :

The Discrete Tauberian Theorem: The relations

$$
\sum_{n=0}^{\infty} G_{n} \xi^{n} \sim(1-\xi)^{-\kappa} L\left(\frac{1}{1-\xi}\right) \quad \text { as } \quad \xi \rightarrow 1^{-}
$$

and

$$
G_{n} \sim \frac{n^{\kappa-1}}{\Gamma(\kappa)} L(n) \quad \text { as } n \rightarrow \infty
$$


are equivalent, provided that $\kappa>0 ; G_{n}>0$ (for large enough $\left.n\right)$; the sequence $\left\{G_{n}\right\}$ is monotonic (for large enough $n$ ); and the function $L$ is slowly varying in the sense that $\frac{L(\lambda x)}{L(x)} \rightarrow 1$ as $x \rightarrow \infty$ for each fixed, positive $\lambda$.

\section{Properties of the lattice walk performed by the tagged particle.}

\subsection{The probability that the tagged particle suffers $k$ encounters during $n$ steps of the vacancy}

Let us denote by $\hat{\pi}_{n}^{k}\left(\mathbf{r}_{\mathbf{0}}\right)$ the probability that the tagged particle has received $k$ hits during $n$ steps of the vacancy, given that the vacancy commenced at site $\mathbf{r}_{\mathbf{0}}$ $\left(\mathbf{r}_{\mathbf{0}} \neq 0\right)$. One can certainly write:

$$
\hat{\pi}_{n}^{k}\left(\mathbf{r}_{\mathbf{0}}\right)=\sum_{\rho} \sum_{\mathbf{r}}^{\prime} \pi_{n}^{k}\left(\boldsymbol{\rho}, \mathbf{r} \mid \mathbf{0}, \mathbf{r}_{\mathbf{0}}\right)
$$

so that the corresponding generating function $\hat{\Pi}^{k}\left(\mathbf{r}_{\mathbf{0}} ; \xi\right)=\sum_{n=0}^{\infty} \hat{\pi}_{n}^{k}\left(\mathbf{r}_{\mathbf{0}}\right) \xi^{n}$ becomes

$$
\hat{\Pi}^{k}\left(\mathbf{r}_{\mathbf{0}} ; \xi\right)=\sum_{\rho} \sum_{\mathbf{r}}{ }^{\prime} \Pi^{k}\left(\boldsymbol{\rho}, \mathbf{r} \mid \mathbf{0}, \mathbf{r}_{\mathbf{0}} ; \xi\right) .
$$

Inserting the expressions (27),(28) into (33) and then employing (23),(22) and (25), we can perform the sums over $\mathbf{r}$ and the $\boldsymbol{\nu}_{j}$-s. The results are

$k \geq 1$ :

$$
\hat{\Pi}^{k}\left(\mathbf{r}_{\mathbf{0}} ; \xi\right)=\frac{1}{1-\xi}\left(1-\frac{u}{t}\right)\left(\frac{u}{t}\right)^{k-1} \frac{P\left(\mathbf{0} \mid \mathbf{r}_{\mathbf{0}} ; \xi\right)}{t},
$$

$k=0$ :

$$
\hat{\Pi}^{0}\left(\mathbf{r}_{\mathbf{0}} ; \xi\right)=\frac{1}{1-\xi}\left[1-\frac{1}{t} P\left(\mathbf{0} \mid \mathbf{r}_{\mathbf{0}} ; \xi\right)\right] .
$$

Eq. (35) is the generating function for the probability $\hat{\pi}_{n}^{0}\left(\mathbf{r}_{\mathbf{0}}\right)$, that the vacancy avoids the origin during the first $n$ steps. For $k \geq 1$, the explicit evaluation of the inverse transform (3) is possible only in one dimension (see Appendix B for the expressions of the corresponding generating functions). However, we do not find these results particularly illuminating and will not present it here. Instead, we analyze these probabilities in the $n \rightarrow \infty$ limit, giving the leading behaviour in $n$ through The Discrete Tauberian Theorem.

$\underline{d=1}$ Using the expressions for the generating functions in $d=1$ from Appendix B and applying the Discrete Tauberian Theorem for $k \geq 1$ with $\kappa=1 / 2$, and for $k=0$ with $\kappa=1$, we find

$$
\hat{\pi}_{n}^{k}\left(\mathbf{r}_{\mathbf{0}}\right) \sim \frac{1}{\sqrt{n}} \sqrt{\frac{2}{\pi}} e^{-\sqrt{2} \frac{k+\left|\mathbf{r}_{\mathbf{0}}\right|-1}{\sqrt{n}}} \text { and for } k=0: \hat{\pi}_{n}^{0}\left(\mathbf{r}_{\mathbf{0}}\right) \sim 1-e^{-\sqrt{2} \frac{\left|\mathbf{r}_{\mathbf{0}}\right|}{\sqrt{n}}}
$$

$\underline{d=2}$ For simplicity, we shall treat here the case $\mathbf{r}_{\mathbf{0}}=\boldsymbol{\alpha}$, only, i.e., the vacancy starts from a nearest neighbour of the tagged particle which is in the origin. As we will see, the probability for the tracer to suffer $k$ hits scales as $\ln n$. Thus we may 
expect that, apart from the initial "wait" until the walker reaches the tracer for the first time (i.e., if $\mathbf{r}_{\mathbf{0}}$ is not necessarily a nearest neighbour), the leading behaviour to be independent of which direction the vacancy first hits the tagged particle.

Thus, we take $P\left(\mathbf{0} \mid \mathbf{r}_{\mathbf{0}} ; \xi\right)=u$. Considering the limiting behaviour (B.10) for $t$ and applying the Tauberian Theorem with $\kappa=1$, we arrive at:

$$
\hat{\pi}_{n}^{k}(\boldsymbol{\alpha}) \sim \frac{\pi}{\ln n} e^{-\pi \frac{k}{\ln n}}, \quad k \geq 0, \quad n \gg 1 .
$$

this result shows that the number of hits the tagged particle received scales like the $\ln n$, with an exponential (or geometric) scaling function. Note that, from Section 4.2 , distances for the Brownian vacancy driven walk do scale as the square root of $k$, the number of steps taken by the tagged particle! Of course, the scaling function there is not Gaussian.

$\underline{d \geq 3} t(1)$ is no longer divergent, but is a finite number larger than 1 . One can easily see that for ' $n=\infty$ ':

$$
\hat{\pi}_{\infty}^{k}\left(\mathbf{r}_{\mathbf{0}}\right)=\frac{1}{t(1)}\left[1-\frac{1}{t(1)}\right]^{k} \frac{P\left(\mathbf{0} \mid \mathbf{r}_{\mathbf{0}} ; 1\right)}{t(1)-1} .
$$

If $\mathbf{r}_{\mathbf{0}}$ is a nearest neighbour of the origin, then $\hat{\pi}_{\infty}^{k}(\boldsymbol{\alpha})=\frac{1}{t(1)}\left[1-\frac{1}{t(1)}\right]^{k}$. If $R(\mathbf{0})$ denotes the probability of return to the origin, then according to Ref. 6 $R(\mathbf{0})=1-1 / t(1)$, thus $\hat{\pi}_{\infty}^{k}(\boldsymbol{\alpha})=[1-R(\mathbf{0})] R(\mathbf{0})^{k}$. Observe that, independently of dimensionality, the large $k$-behaviour is a decaying exponential.

Another interesting question is: "what is the average number of hits suffered by the tagged particle during $n$-steps of the walker i.e., $\langle k\rangle_{n}\left(\mathbf{r}_{\mathbf{0}}\right)^{\prime}$ '? Naively, one expects that it is simply the average number of visits the vacancy pays to the origin, when started from site $\mathbf{r}_{\mathbf{0}}:\langle I\rangle_{n}\left(\mathbf{r}_{\mathbf{0}}\right)$. However, on closer examination, we see that the tagged particle wanders away from its original position (the origin) and towards the initial position of the walker, so that it is not quite at the origin all the time. Therefore, $\langle k\rangle_{n}\left(\mathbf{r}_{\mathbf{0}}\right)$ can differ from $\langle I\rangle_{n}\left(\mathbf{r}_{\mathbf{0}}\right)$. We shall compute the difference $\langle k\rangle_{n}\left(\mathbf{r}_{\mathbf{0}}\right)-\langle I\rangle_{n}\left(\mathbf{r}_{\mathbf{0}}\right)$. This is easily given as:

$$
\langle k\rangle_{n}\left(\mathbf{r}_{\mathbf{0}}\right)-\langle I\rangle_{n}\left(\mathbf{r}_{\mathbf{0}}\right)=\frac{1}{2 \pi \mathrm{i}} \oint_{\Gamma} \frac{d \xi}{\xi^{n+1}} \frac{t-1}{1-(1-\xi) t} P\left(\mathbf{0} \mid \mathbf{r}_{\mathbf{0}} ; \xi\right) .
$$

In one dimension, the large $n$-behaviour turns out to be:

$$
\langle k\rangle_{n}\left(\mathbf{r}_{\mathbf{0}}\right)-\langle I\rangle_{n}\left(\mathbf{r}_{\mathbf{0}}\right) \sim \frac{1}{2}\left(1+\frac{1}{\sqrt{2 n}}\right)\left(1-\sqrt{\frac{2}{n}}\right)^{\left|\mathbf{r}_{\mathbf{0}}\right|+1},
$$

i.e., in the $n \rightarrow \infty$ limit the tagged particle receives 0.5 more visits than the origin.

In two dimensions, after evaluating the cut in the integral (39) in leading order, the following behaviour is found:

$$
\langle k\rangle_{n}\left(\mathbf{r}_{\mathbf{0}}\right)-\langle I\rangle_{n}\left(\mathbf{r}_{\mathbf{0}}\right) \sim \frac{2}{\pi^{2}} \frac{1}{n}[\psi(n)+c] \sim \frac{2}{\pi^{2}} \frac{\ln n}{n}, \quad n \gg 1,
$$


where $\psi(n)$ is the Euler psi-function and $c$ is the Euler constant. After taking the limit $n \rightarrow \infty$, one has $\langle k\rangle_{\infty}\left(\mathbf{r}_{\mathbf{0}}\right)=\langle I\rangle_{\infty}\left(\mathbf{r}_{\mathbf{0}}\right)$.

In $d \geq 3$, there are no singularities in the integrand and the difference approaches to zero rapidly with $n$.

Observe that $\langle k\rangle_{n}\left(\mathbf{r}_{\mathbf{0}}\right)>\langle I\rangle_{n}$, i.e., the tagged particle receives more visits than the origin in any dimension. This is explained by the following. As it will be shown in Section 4.3, the center of the walk performed by the tagged particle is always shifted from origin towards $\mathbf{r}_{\mathbf{0}}$ (by an amount $\langle\boldsymbol{\rho}\rangle_{n}\left(\mathbf{r}_{\mathbf{0}}\right)$ ). Since $\mathbf{r}_{\mathbf{0}}$ is the center of the walk performed by the vacancy, it means that the average position of the tagged particle is always in between the average position of the vacancy and the origin. Thus, the tagged particle receives more visits than the origin.

\subsection{The probability that the tagged particle arrives at site $\rho$ on the $n$-th step of the vacancy}

Let us denote by $\bar{\pi}_{n}\left(\boldsymbol{\rho} \mid \mathbf{r}_{\mathbf{0}}\right)$ the probability that the tagged particle arrives at site $\boldsymbol{\rho}$ on the $n$-th step of the vacancy. Clearly, we have

$$
\bar{\pi}_{n}\left(\boldsymbol{\rho} \mid \mathbf{r}_{\mathbf{0}}\right)=\sum_{k=0}^{\infty} \sum_{\mathbf{r}}^{\prime} \pi_{n}^{k}\left(\boldsymbol{\rho}, \mathbf{r} \mid \mathbf{0}, \mathbf{r}_{\mathbf{0}}\right) .
$$

while the corresponding generating function is

$$
\bar{\Pi}\left(\boldsymbol{\rho} \mid \mathbf{r}_{\mathbf{0}} ; \xi\right)=\sum_{k=0}^{\infty} \sum_{\mathbf{r}}^{\prime} \Pi^{k}\left(\boldsymbol{\rho}, \mathbf{r} \mid \mathbf{0}, \mathbf{r}_{\mathbf{0}} ; \xi\right) .
$$

In this sum, the $k=0$ term has to be treated separately, and it is:

$$
\delta_{\rho, \mathbf{0}} \frac{1}{1-\xi}\left[1-\frac{1}{t} P\left(\mathbf{0} \mid \mathbf{r}_{\mathbf{0}} ; \xi\right)\right] .
$$

Let us denote by $\bar{\Pi}_{1}\left(\boldsymbol{\rho} \mid \mathbf{r}_{\mathbf{0}} ; \xi\right)$ the rest $(k \geq 1)$ of the terms in (43). Using (23), the sum over $\mathbf{r}$ can readily be performed to give:

$$
\bar{\Pi}_{1}\left(\boldsymbol{\rho} \mid \mathbf{r}_{\mathbf{0}} ; \xi\right)=\frac{1-u / t}{1-\xi} \sum_{k=1}^{\infty}(p \xi)^{k} \sum_{\nu_{1}}^{(\mathbf{0})} \ldots \sum_{\nu_{k}}^{(\mathbf{0})} \delta \sum_{\rho, \sum_{j=1}^{k} \nu_{j}} P^{\dagger}\left(\boldsymbol{\nu}_{k} \mid-\boldsymbol{\nu}_{k-1} ; \xi\right) \ldots P^{\dagger}\left(\boldsymbol{\nu}_{1} \mid \mathbf{r}_{\mathbf{0}} ; \xi\right)
$$

After introducing the function

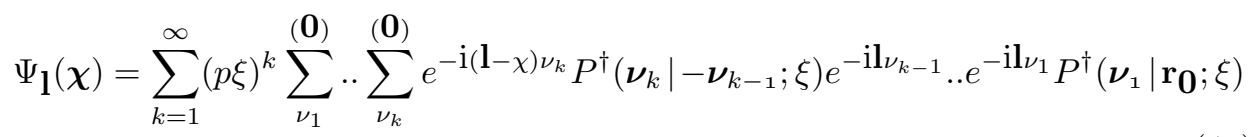

and using the integral representation of the Kronecker delta, one finds:

$$
\bar{\Pi}_{1}\left(\boldsymbol{\rho} \mid \mathbf{r}_{\mathbf{0}} ; \xi\right)=\frac{1-u / t}{1-\xi} \int_{-\pi}^{\pi} \frac{d^{d} l}{(2 \pi)^{d}} e^{-\mathrm{i} \mathbf{l}_{\rho}} \Psi_{\mathbf{l}}(\mathbf{0}) .
$$


Let us now evaluate the function $\Psi_{1}(\chi)$, regarding $\mathbf{l}$ as a parameter. Define first the following integral operator:

$$
\hat{\Gamma}_{\mathbf{l}}^{(\chi)}(\boldsymbol{\vartheta}) \equiv \int_{-\pi}^{\pi} \frac{d^{d} \vartheta}{(2 \pi)^{d}} \frac{\xi \omega(\boldsymbol{\vartheta}-\mathbf{l}+\boldsymbol{\chi})}{1-\xi \omega(\boldsymbol{\vartheta})}
$$

The result of the action of $\hat{\Gamma}_{1}^{(\chi)}(\boldsymbol{\vartheta})$ on a function of variable $\boldsymbol{\vartheta}$ is a function of a variable $\chi$ and parameter $\mathbf{l}$. The $k=1$ term in (46) is

$$
\hat{\Gamma}_{\mathbf{l}}^{(\chi)}(\boldsymbol{\vartheta}) A\left(\mathbf{r}_{\mathbf{0}} ; \xi, \boldsymbol{\vartheta}\right),
$$

where

$$
A\left(\mathbf{r}_{\mathbf{0}} ; \xi, \boldsymbol{\vartheta}\right)=e^{-\mathrm{i} \vartheta \mathbf{r}_{\mathbf{0}}}-\frac{1}{t} P\left(\mathbf{0} \mid \mathbf{r}_{\mathbf{0}} ; \xi\right) .
$$

After performing the sum over $\boldsymbol{\nu}_{k}$ in the rest of the terms $(k \geq 2)$, and comparing the resultant sums with (46), we find the following integral equation:

$$
\Psi_{\mathbf{l}}(\chi)=\hat{\Gamma}_{\mathbf{l}}^{(\chi)}\left(\boldsymbol{\vartheta}_{1}\right) A\left(\mathbf{r}_{\mathbf{0}} ; \xi, \boldsymbol{\vartheta}_{1}\right)-\frac{u}{t} \Psi_{\mathbf{l}}(\mathbf{0}) \hat{\Gamma}_{\mathbf{l}}^{(\chi)}+\hat{\Gamma}_{\mathbf{l}}^{(\chi)}\left(\boldsymbol{\vartheta}_{1}\right) \Psi_{\mathbf{l}}\left(\boldsymbol{\vartheta}_{1}\right),
$$

where $\Gamma_{\mathbf{l}}^{(\chi)}$ is just $\hat{\Gamma}_{\mathbf{l}}^{(\chi)}\left(\boldsymbol{\vartheta}_{1}\right)$ applied on the constant $(=1)$ function. Substituting the right hand side of (51) into the $\Psi_{\mathbf{l}}\left(\boldsymbol{\vartheta}_{1}\right)$ on the right repeatedly, we arrive at:

$$
\Psi_{\mathbf{l}}(\chi)=\hat{T}_{\mathbf{l}}^{(\chi)}\left(\boldsymbol{\vartheta}_{\mathbf{1}}\right) \hat{\Gamma}_{\mathbf{l}}^{\left(\vartheta_{1}\right)}(\boldsymbol{\vartheta}) A\left(\mathbf{r}_{\mathbf{0}} ; \xi, \boldsymbol{\vartheta}\right)-\frac{u}{t} \Psi_{\mathbf{l}}(\mathbf{0}) \hat{T}_{\mathbf{l}}^{(\chi)}\left(\boldsymbol{\vartheta}_{1}\right) \Gamma_{\mathbf{l}}^{\left(\vartheta_{1}\right)},
$$

where

$$
\begin{aligned}
\hat{T}_{\mathbf{l}}^{(\chi)}\left(\boldsymbol{\vartheta}_{1}\right) \equiv \hat{1}^{(\chi)}\left(\boldsymbol{\vartheta}_{1}\right)+\hat{\Gamma}_{\mathbf{l}}^{(\chi)}\left(\boldsymbol{\vartheta}_{1}\right) & +\hat{\Gamma}_{\mathbf{l}}^{(\chi)}\left(\boldsymbol{\vartheta}_{2}\right) \hat{\Gamma}_{\mathbf{l}}^{\left(\vartheta_{2}\right)}\left(\boldsymbol{\vartheta}_{1}\right) \\
& +\hat{\Gamma}_{\mathbf{l}}^{(\chi)}\left(\boldsymbol{\vartheta}_{3}\right) \hat{\Gamma}_{\mathbf{l}}^{\left(\vartheta_{3}\right)}\left(\boldsymbol{\vartheta}_{2}\right) \hat{\Gamma}_{\mathbf{l}}^{\left(\vartheta_{2}\right)}\left(\boldsymbol{\vartheta}_{1}\right) \ldots,
\end{aligned}
$$

with

$$
\hat{1}^{(\chi)}\left(\boldsymbol{\vartheta}_{1}\right) \equiv \int_{-\pi}^{\pi} \frac{d^{d} \vartheta_{1}}{(2 \pi)^{d}} \delta\left(\boldsymbol{\vartheta}_{1}-\chi\right)
$$

Choosing $\chi=0$ in (52):

$$
\Psi_{\mathbf{l}}(\mathbf{0})=\frac{\hat{T}_{\mathbf{l}}^{(\mathbf{0})}\left(\boldsymbol{\vartheta}_{\mathbf{1}}\right) \hat{\Gamma}_{\mathbf{l}}^{\left(\vartheta_{1}\right)}(\boldsymbol{\vartheta}) A\left(\mathbf{r}_{\mathbf{0}} ; \xi, \boldsymbol{\vartheta}\right)}{1+\frac{u}{t} \hat{T}_{\mathbf{l}}^{(\mathbf{0})}\left(\boldsymbol{\vartheta}_{1}\right) \hat{\Gamma}_{\mathbf{l}}^{\left(\vartheta_{1}\right)}} .
$$

In the following we investigate the operators $\hat{\Gamma}$ and $\hat{T}$. By virtue of (12) and (48), the action of $\hat{\Gamma}$ on a function $f: \mathbb{R}^{d} \rightarrow \mathscr{C}$ may be written as:

$$
\hat{\Gamma}_{\mathbf{l}}^{(\chi)}(\boldsymbol{\vartheta}) f(\boldsymbol{\vartheta})=p \xi \sum_{\beta}^{(\mathbf{0})} e^{\mathrm{i} \chi \beta} \int_{-\pi}^{\pi} \frac{d^{d} \vartheta}{(2 \pi)^{d}} \frac{e^{\mathrm{i}(\vartheta-\mathbf{l}) \beta}}{1-\xi \omega(\boldsymbol{\vartheta})} f(\boldsymbol{\vartheta}) \equiv \sum_{\beta}^{(\mathbf{0})} f_{\mathbf{l}_{\beta}} e^{\mathrm{i} \chi \beta}
$$


with $f_{\mathbf{l}_{\beta}}$ as $2 d$, l-dependent complex numbers.

Since the $\boldsymbol{\beta}$ 's are lattice sites (elements of $Z^{d}$ ), they are of the form:

$$
\begin{array}{rrr}
\boldsymbol{\beta}=(0, \ldots 0, & \begin{aligned}
\sigma \\
\hat{\mu}
\end{aligned} & , 0, \ldots, 0), \quad \sigma \in\{-1,1\} \\
& , \quad \mu \in\{1,2, \ldots, d\}
\end{array}
$$

( $\mu$ labels the axes). Then, instead of $\boldsymbol{\beta}$ one can use the integer $j$ given by the following one-to-one correspondence.

$$
j=(1-\sigma) \frac{d}{2}+\mu
$$

if $\boldsymbol{\beta}$ is known. Conversely,

$$
\sigma=1-2\left[\frac{j-1}{d}\right], \quad \mu=j-d\left[\frac{j-1}{d}\right]
$$

when $j$ is given. An immediate consequence is that when $\sigma=+1$, we have $j \in$ $\{1, \ldots, d\}$ and when $\sigma=-1$, we have $j \in\{d+1, \ldots, 2 d\}$.

Let us denote by $V\left(\mathbb{R}^{d}\right)$ the set of functions $v: \mathbb{R}^{d} \rightarrow \mathbb{C}$ spanned by the basis:

$$
\tilde{V}\left(\mathbb{R}^{d}\right)=\left\{b^{1}, b^{2}, \ldots, b^{2 d}\right\}, \quad b^{j}: \mathbb{R}^{d} \rightarrow \mathscr{C}, \quad b^{j}(\chi)=e^{\mathrm{i} \chi \beta(j)},
$$

where $\boldsymbol{\beta}(j)$ is the nearest neighbor of the origin corresponding to the integer $j$ via (59) and (57). $\left(V\left(\mathbb{R}^{d}\right)\right.$ forms a linear space with the usual rules of addition and multiplication of functions over the division ring of the complex numbers.) Note from (56) that the result of the action of $\hat{\Gamma}$ on any function $f: \mathbb{R}^{d} \rightarrow \mathbb{C}$ is a function from $V\left(\mathbb{R}^{d}\right)$. Furthermore, since the operators $\hat{\Gamma}$ and $\hat{T}$ are linear operators they admit a representation by $2 d \times 2 d$ matrices in this linear space with the basis as in (60). Let $\boldsymbol{\Gamma}_{\mathbf{1}}$ and $\mathbf{T}_{\mathbf{l}}$ denote the two matrices. Every element $v=\sum_{j=1}^{2 d} v_{j} b^{j}$ is represented as a $2 d \times 1$ columnar matrix $[v]$ in this basis:

$$
[v]=\left[\begin{array}{c}
v_{1} \\
\cdot \\
\cdot \\
\cdot \\
v_{2 d}
\end{array}\right]
$$

Let us now define the matrix $\mathbf{P}=\left[p_{j}^{m}\right], j, m \in\{1, \ldots, 2 d\}$ in this linear space, with elements given by:

$$
p_{j}^{m}=P(\boldsymbol{\beta}(j) \mid-\boldsymbol{\alpha}(m) ; \xi) .
$$


Explicitly:

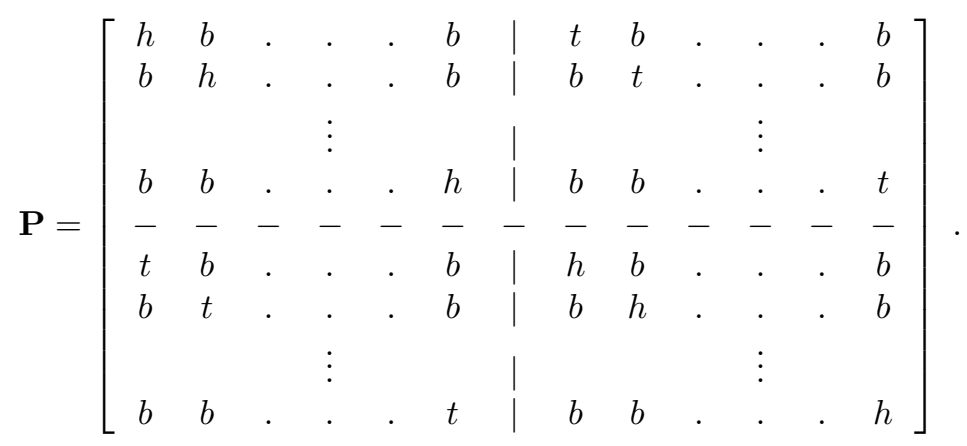

Next, let us consider the diagonal matrix $\mathbf{L}_{\mathbf{l}}=\left[L_{\mathbf{l}_{j}}^{m}\right]$, with matrix elements:

$$
L_{\mathbf{l}_{j}}^{m}=e^{-\mathrm{i} \mathbf{l}_{\beta(j)}} \delta_{j}^{m},
$$

where $\delta_{j}^{m}$ (Kronecker delta) are the elements of the $2 d \times 2 d$ unit matrix 1 .

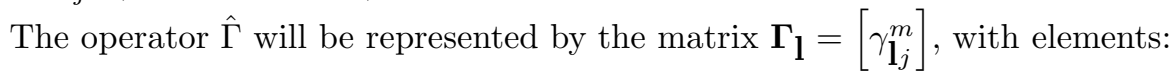

$$
\gamma_{\mathbf{l} j}^{m}=p \xi \int_{-\pi}^{\pi} \frac{d^{d} \vartheta}{(2 \pi)^{d}} \frac{e^{\mathrm{i} \vartheta[\alpha(m)+\beta(j)]}}{1-\xi \omega(\boldsymbol{\vartheta})} e^{-\mathrm{i} \mathbf{l} \beta(j)}=p \xi P(\boldsymbol{\beta}(j) \mid-\boldsymbol{\alpha}(m) ; \xi) e^{-\mathrm{i} \mathbf{l} \beta(j)}
$$

From the above, we have:

$$
\boldsymbol{\Gamma}_{\mathbf{l}}=p \xi \mathbf{L}_{\mathbf{l}} \mathbf{P} .
$$

Then, based on (53), it is easy to write down the representation for the operator $\hat{T}$, $\mathbf{T}_{\mathbf{l}}=\left[t_{\mathbf{l}_{j}}^{m}\right]$ :

$$
\mathbf{T}_{\mathbf{l}}=\mathbf{1}+\boldsymbol{\Gamma}_{\mathbf{l}}+\boldsymbol{\Gamma}_{\mathbf{l}}^{2}+\Gamma_{1}^{3}+\ldots=\left(\mathbf{1}-\boldsymbol{\Gamma}_{\mathbf{l}}\right)^{-1} .
$$

Examining (55), it is obvious that we need to calculate the action of the operator $\hat{\Gamma}$ on two functions: the constant $(=1)$ function and $e^{-\mathbf{i} \vartheta \mathbf{r}} \mathbf{0}$. If $\left[\iota_{\mathbf{l}}\right]$ denotes the vector representing the action of $\hat{\Gamma}$ on the function unity and $\left[\tau_{1}\right]$ represents the action of $\hat{\Gamma}$ on $e^{-\mathrm{i} \vartheta \mathbf{r}} \mathbf{0}$, we have:

$$
\begin{aligned}
& {\left[\iota_{\mathbf{l}}\right]=\sqrt{p} \xi u \mathbf{L}_{\mathbf{l}}\left[\phi_{1}\right],} \\
& {\left[\tau_{\mathbf{l}}\right]=p \xi \mathbf{L}_{\mathbf{l}}\left[Y_{0}\right]}
\end{aligned}
$$

where $\left[\phi_{1}\right]$ is the eigenvector of $\mathbf{P}$ corresponding to the eigenvalue $\lambda_{1}=u /(p \xi)$ (see Appendix $\mathrm{C}$ ) and $\left[Y_{0}\right]$ is the vector:

$$
\left[Y_{0}\right]=\left[\begin{array}{c}
P\left(\boldsymbol{\beta}(1) \mid \mathbf{r}_{\mathbf{0}} ; \xi\right) \\
\vdots \\
P\left(\boldsymbol{\beta}(2 d) \mid \mathbf{r}_{\mathbf{0}} ; \xi\right)
\end{array}\right]
$$


Note that

$$
\left[\phi_{1}\right]^{T}\left[Y_{0}\right]=\frac{1}{\sqrt{p} \xi} P\left(\mathbf{0} \mid \mathbf{r}_{\mathbf{0}} ; \xi\right)
$$

Since

$$
\begin{aligned}
\hat{T}_{\mathbf{l}}^{(\chi)}\left(\boldsymbol{\vartheta}_{1}\right) \Gamma_{\mathbf{l}}^{\left(\vartheta_{1}\right)} & =\sum_{j=1}^{2 d}\left(\mathbf{T}_{\mathbf{l}}\left[\iota_{\mathbf{l}}\right]\right)_{j} b^{j}(\chi), \\
\hat{T}_{\mathbf{l}}^{(\chi)}\left(\boldsymbol{\vartheta}_{\mathbf{1}}\right) \hat{\Gamma}_{\mathbf{l}}^{\left(\vartheta_{1}\right)}(\boldsymbol{\vartheta}) e^{-\mathrm{i} \vartheta \mathbf{r}_{\mathbf{0}}} & =\sum_{j=1}^{2 d}\left(\mathbf{T}_{\mathbf{l}}\left[\tau_{\mathbf{l}}\right]\right)_{j} b^{j}(\boldsymbol{\chi}),
\end{aligned}
$$

and $b^{j}(0)=1$, we arrive at:

$$
\Psi_{\mathbf{l}}(\mathbf{0})=\frac{\sqrt{p} \xi\left[\phi_{1}\right]^{T} \mathbf{T}_{\mathbf{l}} \mathbf{L}_{\mathbf{l}}\left[Y_{0}\right]-\xi \frac{u}{t} P\left(\mathbf{0} \mid \mathbf{r}_{\mathbf{0}} ; \xi\right)\left[\phi_{1}\right]^{T} \mathbf{T}_{\mathbf{l}} \mathbf{L}_{\mathbf{l}}\left[\phi_{1}\right]}{1+\xi \frac{u^{2}}{t}\left[\phi_{1}\right]^{T} \mathbf{T}_{\mathbf{l}} \mathbf{L}_{\mathbf{l}}\left[\phi_{1}\right]} .
$$

In Appendix C, we show how to diagonalise the matrix $\mathbf{P}$ and write it in the form $\mathbf{P}=\mathbf{C}^{-1} \boldsymbol{\Omega} \mathbf{C}$, with $\boldsymbol{\Omega}$ a diagonal matrix containing the eigenvalues of $\mathbf{P}$ on the diagonal. The columns of the matrix $\mathbf{C}^{-1}$ are the right eigenvectors of $\mathbf{P}$. Therefore

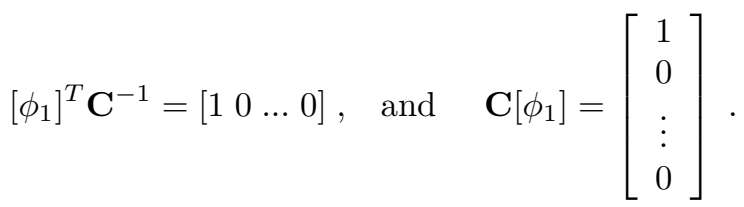

Thus

$$
\begin{aligned}
& {\left[\phi_{1}\right]^{T} \mathbf{T}_{\mathbf{l}} \mathbf{L}_{\mathbf{l}}\left[\phi_{1}\right]=\left[\phi_{1}\right]^{T}\left(\mathbf{1}-p \xi \mathbf{L}_{\mathbf{l}} \mathbf{P}\right)^{-1} \mathbf{L}_{\mathbf{l}}\left[\phi_{1}\right]=\left[\phi_{1}\right]^{T}\left(\mathbf{L}_{-} \mathbf{l}-p \xi \mathbf{P}\right)^{-1}\left[\phi_{1}\right]=} \\
& {\left[\phi_{1}\right]^{T} \mathbf{C}^{-1}\left(\mathbf{C L}_{-\mathbf{l}} \mathbf{C}^{-1}-p \xi \boldsymbol{\Omega}\right)^{-1} \mathbf{C}\left[\phi_{1}\right],}
\end{aligned}
$$

where we have used the fact $\mathbf{L}_{\mathbf{l}}^{-1}=\mathbf{L}_{-\mathbf{l}}$. Therefore $\left[\phi_{1}\right]^{T} \mathbf{T}_{\mathbf{l}} \mathbf{L}_{\mathbf{l}}\left[\phi_{1}\right]$ is nothing but the $m=1, j=1$ element of the matrix $\left(\mathbf{C L}_{-1} \mathbf{C}^{-1}-p \xi \boldsymbol{\Omega}\right)^{-1}$.

For $l=0$, we have $\mathbf{L}_{0}=\mathbf{1}$, thus the first element in the above matrix is:

$$
\left[\phi_{1}\right]^{T} \mathbf{T}_{0} \mathbf{L}_{0}\left[\phi_{1}\right]=\frac{1}{1-p \xi \lambda_{1}}=\frac{1}{1-u} .
$$

With the help of (11), the first term in the numerator of (74) becomes:

$$
\sqrt{p} \xi\left[\phi_{1}\right]^{T} \mathbf{T}_{0} \mathbf{L}_{0}\left[Y_{0}\right]=\frac{1}{1-u} \frac{1}{t} P\left(\mathbf{0} \mid \mathbf{r}_{\mathbf{0}} ; \xi\right),
$$

and the second:

$$
\xi \frac{u}{t} P\left(\mathbf{0} \mid \mathbf{r}_{\mathbf{0}} ; \xi\right)\left[\phi_{1}\right]^{T} \mathbf{T}_{0} \mathbf{L}_{0}\left[\phi_{1}\right]=\xi \frac{u}{t} \frac{1}{1-u} P\left(\mathbf{0} \mid \mathbf{r}_{\mathbf{0}} ; \xi\right) .
$$

Collecting all the above together, we have:

$$
\Psi_{0}(0)=\frac{1}{1-\frac{u}{t}} \frac{1}{t} P\left(\mathbf{0} \mid \mathbf{r}_{\mathbf{0}} ; \xi\right) .
$$


One check for the above calculations is to see if the distribution (42) is normalized. For this purpose, we consider:

$$
\begin{aligned}
& \sum_{\rho} \bar{\Pi}\left(\boldsymbol{\rho} \mid \mathbf{r}_{\mathbf{0}} ; \xi\right)=\frac{1}{1-\xi}\left[1-\frac{1}{t} P\left(\mathbf{0} \mid \mathbf{r}_{\mathbf{0}} ; \xi\right)\right]+\sum_{\rho} \bar{\Pi}_{1}\left(\boldsymbol{\rho} \mid \mathbf{r}_{\mathbf{0}} ; \xi\right)= \\
& \frac{1}{1-\xi}\left[1-\frac{1}{t} P\left(\mathbf{0} \mid \mathbf{r}_{\mathbf{0}} ; \xi\right)\right]+\frac{1-u / t}{1-\xi} \Psi_{0}(0)=\frac{1}{1-\xi},
\end{aligned}
$$

showing that normalization is indeed properly satisfied.

Considering the first term (44) and the expression (79), the generating function is:

$$
\bar{\Pi}\left(\boldsymbol{\rho} \mid \mathbf{r}_{\mathbf{0}} ; \xi\right)=\frac{1}{1-\xi} \int_{-\pi}^{\pi} \frac{d^{d} l}{(2 \pi)^{d}} e^{-\mathrm{i} \mathbf{l}_{\rho}}\left\{1+\left(1-\frac{u}{t}\right)\left[\Psi_{\mathbf{l}}(\mathbf{0})-\Psi_{\mathbf{0}}(\mathbf{0})\right]\right\},
$$

where $\Psi_{\mathbf{l}}(\mathbf{0})$ is given by (74).

In the following we examine the meaning of formulas (81) and (74), by considering them in different spatial dimensions.

$\underline{d=1}$. This case is considered more as a check for the validity of our formulas since in one dimensions the problem is rather trivial: the random walker is either on the left or on the right of the tagged particle, and the only way to change sides is to exchange positions with the tagged particle. So, if initially the tagged particle was in the origin and the walker was to the right $\left(r_{0}>0\right)$, the only two sites visited by the tagged particle are sites 0 and 1 . Nevertheless, one can ask a nontrivial question: 'what is the probability to find the tagged particle on sites 0 and 1 after $n$-steps of the random walker, given the walker started from site $r_{0}$ ?' This can be answered in the framework of our theory. Without loss of generality, we assume $r_{0}>0$.

The matrix $\mathbf{P}$ is in the simplest possible form:

$$
\mathbf{P}=\left[\begin{array}{cc}
h & t \\
t & h
\end{array}\right]
$$

The computation of $\Psi_{\mathbf{l}}(\mathbf{0})$ is rather straightforward using (74) :

$$
\Psi_{l}(0)=\Psi_{0}(0)\left(1-\frac{1-e^{-\mathrm{i} l}}{1+\frac{u}{t}}\right),
$$

where $\Psi_{0}(0)$ is given by $(79)$ and

$$
P\left(0 \mid r_{0} ; \xi\right)=u\left(\frac{u}{t}\right)^{r_{0}-1}, \quad \frac{u}{t}=\frac{1-\sqrt{1-\xi^{2}}}{\xi} .
$$

See Appendix B for the generating functions. The integral over $l$ in $(47)$ is trivial and we finally obtain:

$$
\bar{\Pi}\left(\rho \mid r_{0} ; \xi\right)=\frac{1}{1-\xi}\left\{\left[1-\frac{\left(\frac{u}{t}\right)^{r_{0}}}{1+\frac{u}{t}}\right] \delta_{\rho, 0}+\frac{\left(\frac{u}{t}\right)^{r_{0}}}{1+\frac{u}{t}} \delta_{\rho, 1}\right\} .
$$


Indeed, as expected, there are only two sites the tagged particle may visit! Moreover, using Eqs. (3) and (何, we have $\bar{\pi}_{\infty}\left(\rho \mid r_{0}\right)=(1 / 2)\left(\delta_{\rho, 0}+\delta_{\rho, 1}\right)$. In other words, the tagged particle spends half of its time on site 1 and half on site 0 . The $n$-dependence is found by simply performing the integral with respect to $\xi$.

$\underline{d=2}$. This is the nontrivial case. In the theory of recurrent events it is shown under very general circumstances that the return probability $R\left(\mathbf{s}_{\mathbf{0}}\right)$ to a site $\mathbf{s}_{\mathbf{0}}$ for a lattice walk, is simply expressed by: $R\left(\mathbf{s}_{\mathbf{0}}\right)=1-1 / P\left(\mathbf{s}_{\mathbf{0}} \mid \mathbf{s}_{\mathbf{0}} ; 1^{-}\right)$, where $P\left(\mathbf{s}_{\mathbf{0}} \mid \mathbf{s}_{\mathbf{0}} ; \xi\right)$ is the generating function for the lattice walk (which can be almost anything, not necessarily Brownian). In Ref. 9 we proved that the same conditions are applicable to the walk performed by the tagged particle as well, provided we replace $P_{n}\left(\mathbf{s} \mid \mathbf{s}_{\mathbf{0}}\right)$ by $\bar{\pi}_{n}\left(\boldsymbol{\rho} \mid \mathbf{r}_{\mathbf{0}}\right)$. Here, $n$ means both 'time' and the number of steps taken by the vacancy, $\boldsymbol{\rho}$ "corresponds" to $\mathbf{s}$ and, according to our initial conditions, $\mathbf{s}_{\mathbf{0}}$ is replaced by $\mathbf{0}$ while $\mathbf{r}_{\mathbf{0}}$ serves as a parameter. Therefore, the walk performed by the tagged particle is recurrent if $\bar{\Pi}\left(\mathbf{o} \mid \mathbf{r}_{\mathbf{0}} ; 1^{-}\right)$is divergent, which is indeed the case, see below.

In two dimensions our matrices are four by four, and the process of evaluating $\bar{\Pi}\left(\boldsymbol{\rho} \mid \mathbf{r}_{\mathbf{0}} ; \xi\right)$ via (81) and (74) is not particularly illuminating. We report only its final, analytic expression:

$$
\bar{\Pi}\left(\boldsymbol{\rho} \mid \mathbf{r}_{\mathbf{0}} ; \xi\right)=\frac{\delta_{\rho, \mathbf{0}}}{1-\xi}\left[1-\frac{1}{t} P\left(\mathbf{0} \mid \mathbf{r}_{\mathbf{0}} ; \xi\right)\right]+\frac{1}{1-\xi} \sum_{j=1}^{4} g_{j}(\boldsymbol{\rho} ; \xi) \mathcal{K}_{j}\left(\mathbf{r}_{\mathbf{0}} ; \xi\right),
$$

where

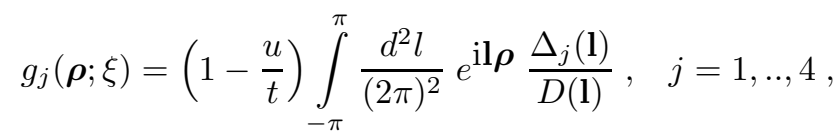

and

$$
\begin{aligned}
\mathcal{K}_{1}\left(\mathbf{r}_{\mathbf{0}} ; \xi\right)= & \frac{1}{t} P\left(\mathbf{0} \mid \mathbf{r}_{\mathbf{0}} ; \xi\right) \\
\mathcal{K}_{2}\left(\mathbf{r}_{\mathbf{0}} ; \xi\right)= & \frac{\xi}{4}\left[P\left(\boldsymbol{\beta}(1) \mid \mathbf{r}_{\mathbf{0}} ; \xi\right)+P\left(-\boldsymbol{\beta}(1) \mid \mathbf{r}_{\mathbf{0}} ; \xi\right)\right. \\
& \left.-P\left(\boldsymbol{\beta}(2) \mid \mathbf{r}_{\mathbf{0}} ; \xi\right)-P\left(-\boldsymbol{\beta}(2) \mid \mathbf{r}_{\mathbf{0}} ; \xi\right)\right], \\
\mathcal{K}_{3}\left(\mathbf{r}_{\mathbf{0}} ; \xi\right)= & -\frac{\xi}{2 \sqrt{2}}\left[P\left(\boldsymbol{\beta}(2) \mid \mathbf{r}_{\mathbf{0}} ; \xi\right)-P\left(-\boldsymbol{\beta}(2) \mid \mathbf{r}_{\mathbf{0}} ; \xi\right)\right], \\
\mathcal{K}_{4}\left(\mathbf{r}_{\mathbf{0}} ; \xi\right)= & \frac{\xi}{2 \sqrt{2}}\left[P\left(\boldsymbol{\beta}(1) \mid \mathbf{r}_{\mathbf{0}} ; \xi\right)-P\left(-\boldsymbol{\beta}(1) \mid \mathbf{r}_{\mathbf{0}} ; \xi\right)\right] .
\end{aligned}
$$

The quantities $\Delta_{j}(\mathbf{l})$ are proportional to the elements (up to a common factor) of the first row of the matrix $\left(\mathbf{C} \mathbf{L}_{-1} \mathbf{C}^{-1}-p \xi \boldsymbol{\Omega}\right)^{-1}$ and they are all expressed in terms of $V=V(\xi)=\frac{1}{\xi}\left[\frac{4}{\pi} \mathbf{E}(\xi)-1-\left(1-\xi^{2}\right) \frac{2}{\pi} \mathbf{K}(\xi)\right]$ and $W=W(\xi)=\frac{1}{\xi}\left[\frac{2}{\pi} \mathbf{E}(\xi)-1\right]$ which are the second and third eigenvalues of matrix $\mathbf{P}, \lambda_{2}$ and $\lambda_{3}$ up to a factor $p \xi=\xi / 4$, Here, $\mathbf{K}(\xi)$ and $\mathbf{E}(\xi)$ being the complete elliptic integrals of first and second kind, 
respectively (see Appendices $\mathrm{C}$ and $\mathrm{B}$ ).

$$
\begin{aligned}
& \Delta_{1}(\mathbf{l})=-(1+V W) W+[(1+V W)+(V+W) W] \frac{1}{2}\left[\cos \left(l_{1}\right)+\cos \left(l_{2}\right)\right] \\
& \Delta_{2}(\mathbf{l})=\left(1-W^{2}\right) \frac{1}{2}\left[\cos \left(l_{1}\right)+\cos \left(l_{2}\right)\right], \\
& \Delta_{3}(\mathbf{l})=\frac{\mathrm{i}}{\sqrt{2}} \sin \left(l_{2}\right)\left[(1+V W)-(V+W) \cos \left(l_{1}\right) \cos \left(l_{2}\right)\right. \\
& \Delta_{4}(\mathbf{l})=-\frac{\mathrm{i}}{\sqrt{2}} \sin \left(l_{1}\right)\left[(1+V W)-(V+W) \cos \left(l_{2}\right)\right]
\end{aligned}
$$

and

$$
\begin{array}{r}
D(\mathbf{l})=(1+V W)\left(1+W \frac{u}{t}\right)\left\{1-(A+B) \frac{1}{2}\left[\cos \left(l_{1}\right)+\cos \left(l_{2}\right)\right]\right. \\
\left.+A B \cos \left(l_{1}\right) \cos \left(l_{2}\right)\right\},
\end{array}
$$

with

$$
A=\frac{W+\frac{u}{t}}{1+W \frac{u}{t}}, \quad B=\frac{V+W}{1+V W} .
$$

Formulas 86-97) give the exact expression of the generating function $\bar{\Pi}\left(\boldsymbol{\rho} \mid \mathbf{r}_{\mathbf{0}} ; \xi\right)$. From (86) we see that the functions $g_{j}$ carry the information on the spatial component of the walk performed by the tagged particle while the functions $\mathcal{K}_{j}$ describe the dependence on the initial condition of the vacancy. Rewriting the cosines and sines in formulas (92- 95) in terms of exponentials, it becomes clear that one needs in general to evaluate the $\xi \rightarrow 1^{-}$behaviour of integrals of type:

$$
\Gamma(\mathbf{s} ; \xi)=\int_{-\pi}^{\pi} \frac{d^{2} l}{(2 \pi)^{2}} \frac{e^{\mathrm{il} \mathbf{s}}}{D(\mathbf{l})} .
$$

By further introducing the vectors

$$
[\Gamma(\boldsymbol{\rho} ; \xi)]_{n .} \equiv\left[\begin{array}{c}
\Gamma(\boldsymbol{\rho}+\boldsymbol{\beta}(1) ; \xi) \\
\Gamma(\boldsymbol{\rho}+\boldsymbol{\beta}(2) ; \xi) \\
\Gamma(\boldsymbol{\rho}-\boldsymbol{\beta}(1) ; \xi) \\
\Gamma(\boldsymbol{\rho}-\boldsymbol{\beta}(2) ; \xi)
\end{array}\right], \quad[\Gamma(\boldsymbol{\rho} ; \xi)]_{n . n .} \equiv\left[\begin{array}{c}
\Gamma(\boldsymbol{\rho}+\boldsymbol{\beta}(1)+\boldsymbol{\beta}(2) ; \xi) \\
\Gamma(\boldsymbol{\rho}-\boldsymbol{\beta}(1)+\boldsymbol{\beta}(2) ; \xi) \\
\Gamma(\boldsymbol{\rho}-\boldsymbol{\beta}(1)-\boldsymbol{\beta}(2) ; \xi) \\
\Gamma(\boldsymbol{\rho}+\boldsymbol{\beta}(1)-\boldsymbol{\beta}(2) ; \xi)
\end{array}\right]
$$

characterizing the nearest neighbour and next to nearest neighbour terms corresponding to site $\boldsymbol{\rho}$, the $g_{j}$ functions are simply expressed as:

$$
\begin{aligned}
g_{1}(\boldsymbol{\rho} ; \xi)=- & (1+V W) W\left(1-\frac{u}{t}\right) \Gamma(\boldsymbol{\rho} ; \xi)+ \\
& \frac{1}{2}[(1+V W)+(V+W) W]\left(1-\frac{u}{t}\right)\left[\phi_{1}\right]^{T}[\Gamma(\boldsymbol{\rho} ; \xi)]_{n .}- \\
& \frac{1}{2}(V+W)\left(1-\frac{u}{t}\right)\left[\phi_{1}\right]^{T}[\Gamma(\boldsymbol{\rho} ; \xi)]_{n . n .} .
\end{aligned}
$$




$$
\begin{aligned}
g_{2}(\boldsymbol{\rho} ; \xi)=- & \frac{1}{2}\left(1-W^{2}\right)\left(1-\frac{u}{t}\right)\left[\phi_{2}\right]^{T}[\Gamma(\boldsymbol{\rho} ; \xi)]_{n .}, \\
g_{3}(\boldsymbol{\rho} ; \xi)=- & \frac{1}{2}(1+V W)\left(1-\frac{u}{t}\right)\left[\phi_{3}\right]^{T}[\Gamma(\boldsymbol{\rho} ; \xi)]_{n .}+ \\
& +\frac{1}{4}(V+W)\left(1-\frac{u}{t}\right)\left(\left[\phi_{3}\right]^{T}+\left[\phi_{4}\right]^{T}\right)[\Gamma(\boldsymbol{\rho} ; \xi)]_{n . n .}, \\
g_{4}(\boldsymbol{\rho} ; \xi)= & \frac{1}{2}(1+V W)\left(1-\frac{u}{t}\right)\left[\phi_{4}\right]^{T}[\Gamma(\boldsymbol{\rho} ; \xi)]_{n .}+ \\
& +\frac{1}{4}(V+W)\left(1-\frac{u}{t}\right)\left(\left[\phi_{4}\right]^{T}-\left[\phi_{3}\right]^{T}\right)[\Gamma(\boldsymbol{\rho} ; \xi)]_{n . n .} .
\end{aligned}
$$

The $\xi \rightarrow 1^{-}$behaviour is determined by the singularities of $D(\mathbf{l})$. Analyzing the functions $A$ and $B$ one can rigurously show that there is only one zero of $D(\mathbf{l})$ in the first Brillouin zone, namely $\mathbf{l}=0$.

For $\mathbf{s}=0,98)$ can be evaluated exactly:

$$
\Gamma(\mathbf{0} ; \xi)=\frac{2}{\pi} \frac{1}{(1+V W)\left(1+W \frac{u}{t}\right)} \frac{1}{1-A B} \mathbf{K}\left(\frac{A-B}{1-A B}\right) .
$$

For $\mathbf{s} \neq 0$ the exact evaluation of the integral is cumbersome (it can be given recursively and in terms of Legendre functions of second kind) and it is not our aim here. Instead we analyze the $\xi \rightarrow 1^{-}$behaviour. In this limit $u / t \rightarrow 1$, $V \rightarrow \bar{V}=\frac{4}{\pi}-1 \sim 0.273, W \rightarrow \bar{W}=\frac{2}{\pi}-1 \sim-0.363, A \rightarrow \bar{A}=1, B \rightarrow \bar{B} \sim-0.100$, see also Appendices B and C. The limiting behaviours for both $\mathbf{s}=0$ and $\mathbf{s} \neq 0$ cases are computed to be:

$$
\Gamma(\mathbf{0} ; \xi) \sim \frac{\pi^{2}}{4} \frac{1}{(\pi-2)(\pi-1)} \mathbf{K}\left(1-\frac{2}{\pi-2}(1-u / t)\right), \quad \xi \rightarrow 1^{-},
$$

and

$$
\Gamma(\mathbf{s} ; \xi) \sim \frac{\pi^{2}}{4} \frac{1}{(\pi-2)(\pi-1)} K_{0}(2 \sqrt{\pi-1}|\mathbf{s}| \sqrt{1-u / t}), \quad \xi \rightarrow 1^{-}, \quad \mathbf{s} \neq 0 .
$$

with $K_{0}$ being a modified Bessel function. Let us now keep $|\mathbf{s}|$ constant. Since : $K_{0}(z) \sim-\ln z$ as $z \rightarrow 0$ and $\mathbf{K}(z) \sim \frac{1}{2} \ln \left(\frac{8}{1-z}\right)$ as $z \rightarrow 1^{-}$it follows that both $\Gamma^{\prime}$ 's diverge and have the same leading term, namely:

$$
\Gamma(\mathbf{s} ; \xi) \sim \frac{\pi^{2}}{8} \frac{1}{(\pi-2)(\pi-1)} \ln \left(\frac{1}{1-\frac{u}{t}}\right), \quad \xi \rightarrow 1^{-} .
$$

Taking into account that, in (101-103), $\left[\phi_{j}\right]^{T}[\Gamma(\boldsymbol{\rho} ; \xi)]_{n}$. and $\left[\phi_{j}\right]^{T}[\Gamma(\boldsymbol{\rho} ; \xi)]_{n . n \text {. for }}$ $j \geq 2$ involve differences of $\Gamma$ 's, we see that the nearest neighbour terms and the next nearest neighbour terms (and therefore $g_{2}, g_{3}, g_{4}$ ) are negligible compared to the terms in $g_{1}$. Thus, to leading order, we have:

$$
g_{1}(\boldsymbol{\rho} ; \xi)=\left(1-\frac{1}{\pi}\right)\left(1-\frac{u}{t}\right) \ln \left(\frac{1}{1-\frac{u}{t}}\right)+\mathcal{O}\left(1-\frac{u}{t}\right), \quad \xi \rightarrow 1^{-},
$$




$$
g_{j}(\boldsymbol{\rho} ; \xi)=0+\mathcal{O}\left(1-\frac{u}{t}\right), \quad \xi \rightarrow 1^{-}, \quad j=2,3,4 .
$$

One can see that the leading one is $g_{1}(\boldsymbol{\rho} ; \xi)$. Although the generating functions $P\left(\mathbf{s} \mid \mathbf{r}_{\mathbf{0}} ; 1^{-}\right)$are divergent in two dimensions, the quantities $\mathcal{K}_{j}\left(\mathbf{r}_{\mathbf{0}} ; 1^{-}\right)$are all finite numbers. This is the consequence of formulas (8891) and the fact that when $\xi \rightarrow 1^{-}$the probability generating functions for the Pólya walk all diverge in the same way, more specifically $\lim _{\xi \rightarrow 1^{-}}\left[t(\xi)-P\left(\mathbf{s} \mid \mathbf{r}_{\mathbf{0}} ; \xi\right)\right]$ is a finite number, for any $\mathbf{s}$ and $\mathbf{r}_{\mathbf{0}}$ (see for e.g. Ref. 6, p.145). Thus $\mathcal{K}_{1}\left(\mathbf{r}_{\mathbf{0}} ; 1^{-}\right)=1$. Using this, it also can be proved that the $k=0$ term (44) is negligible, compared to the $g_{1} \mathcal{K}_{1}$ term, in this limit.

Collecting the above, our conclusion is: when $\rho$ is kept finite, the generating function $\bar{\Pi}\left(\boldsymbol{\rho} \mid \mathbf{r}_{\mathbf{0}} ; \xi\right)$ is, to leading order,

$$
\bar{\Pi}\left(\boldsymbol{\rho} \mid \mathbf{r}_{\mathbf{0}} ; \xi\right) \sim\left(1-\frac{1}{\pi}\right) \frac{1}{1-\xi}\left(1-\frac{u}{t}\right) \ln \left(\frac{1}{1-\frac{u}{t}}\right), \quad \xi \rightarrow 1^{-}, \quad \boldsymbol{\rho} \text { finite } .
$$

Using the Discrete Tauberian Theorem and the fact that:

$$
1-\frac{u}{t} \sim \frac{\pi}{\ln \left(\frac{1}{1-\xi}\right)}, \quad \text { as } 0<1-\xi \ll 1,
$$

we find:

$$
\bar{\pi}_{n}\left(\boldsymbol{\rho} \mid \mathbf{r}_{\mathbf{0}}\right) \sim(\pi-1) \frac{\ln (\ln n)}{\ln n}, \quad n \rightarrow \infty, \quad \boldsymbol{\rho} \text { finite } .
$$

From (110), $\bar{\Pi}\left(\mathbf{0} \mid \mathbf{r}_{\mathbf{0}} ; 1^{-}\right)$is clearly divergent, proving our assertion that the walk in two dimensions is reccurent. The tagged particle is guaranteed to visit any site in the $n \rightarrow \infty$ limit.

Scaling. It is well known that, for the pure random (Pólya) walk, distances scale as the square root of time, while the scaling function is, apart from a prefactor, Gaussian. However, for the Brownian vacancy driven walk, the scaling properties are quite different. When $|\boldsymbol{\rho}|$ and $n$ are both large (keep $\mathbf{r}_{\mathbf{0}}$ constant), the only surviving term is again $g_{1} \mathcal{K}_{1}$. Since $\mathcal{K}_{1}\left(\mathbf{r}_{\mathbf{0}} ; 1^{-}\right)=1$, we infer

$$
\begin{array}{r}
\bar{\Pi}\left(\boldsymbol{\rho} \mid \mathbf{r}_{\mathbf{0}} ; \xi\right) \sim \frac{2}{\pi}(\pi-1) \frac{1}{1-\xi}\left(1-\frac{u}{t}\right) K_{0}(2 \sqrt{\pi-1}|\boldsymbol{\rho}| \sqrt{1-u / t}), \\
\xi \rightarrow 1^{-},|\boldsymbol{\rho}| \gg 1,
\end{array}
$$

or

$$
\bar{\pi}_{n}\left(\boldsymbol{\rho} \mid \mathbf{r}_{\mathbf{0}}\right) \sim 2(\pi-1) \frac{1}{\ln n} K_{0}\left(2 \sqrt{\pi(\pi-1)} \frac{|\boldsymbol{\rho}|}{\sqrt{\ln n}}\right), \quad n \rightarrow \infty,|\boldsymbol{\rho}| \gg 1 .
$$

This is the scaling function in the corresponding limit. As we see, instead of being Gaussian, it is the modified Bessel function $K_{0}$. From its argument, it follows that distances scale like square root of the log of time.

In the following Section we calculate the first and second moments for the displacement of the tagged particle. However, instead of using the distribution 
$\bar{\pi}_{n}\left(\boldsymbol{\rho} \mid \mathbf{r}_{\mathbf{0}}\right)$ derived in this Section, we will use a slightly different, but easier, way to arrive at the desired results.

\subsection{Average displacement and standard deviation of the tagged particle.}

Let us define the first and second moments associated with the tagged particle's displacement $\rho$ up to time $n$ :

$$
\langle\boldsymbol{\rho}\rangle_{n}\left(\mathbf{r}_{\mathbf{0}}\right)=\sum_{\rho} \boldsymbol{\rho} \bar{\pi}_{n}\left(\boldsymbol{\rho} \mid \mathbf{r}_{\mathbf{0}}\right)=\sum_{\rho} \boldsymbol{\rho} \sum_{k=0}^{\infty} \sum_{\mathbf{r}}^{\prime} \pi_{n}^{k}\left(\boldsymbol{\rho}, \mathbf{r} \mid \mathbf{0}, \mathbf{r}_{\mathbf{0}}\right),
$$

and

$$
\left\langle\boldsymbol{\rho}^{2}\right\rangle_{n}\left(\mathbf{r}_{\mathbf{0}}\right)=\sum_{\rho} \boldsymbol{\rho}^{2} \bar{\pi}_{n}\left(\boldsymbol{\rho} \mid \mathbf{r}_{\mathbf{0}}\right)=\sum_{\rho} \boldsymbol{\rho}^{2} \sum_{k=0}^{\infty} \sum_{\mathbf{r}}^{\prime} \pi_{n}^{k}\left(\boldsymbol{\rho}, \mathbf{r} \mid \mathbf{0}, \mathbf{r}_{\mathbf{0}}\right),
$$

respectively. The first of these is, of course, the average displacement for the particle: $\langle\boldsymbol{\rho}\rangle_{n}\left(\mathbf{r}_{\mathbf{0}}\right)$. The second is used in finding the "spread", i.e., the standard deviation:

$$
\mathcal{D}_{n}\left(\mathbf{r}_{\mathbf{0}}\right) \equiv \sqrt{\left\langle\boldsymbol{\rho}^{2}\right\rangle_{n}\left(\mathbf{r}_{\mathbf{0}}\right)-\left[\langle\boldsymbol{\rho}\rangle_{n}\left(\mathbf{r}_{\mathbf{0}}\right)\right]^{2}} .
$$

Instead of computing directly the sums above we turn to generating functions

$$
\mathbf{A}\left(\mathbf{r}_{\mathbf{0}} ; \xi\right) \equiv \sum_{n=0}^{\infty}\langle\boldsymbol{\rho}\rangle_{n}\left(\mathbf{r}_{\mathbf{0}}\right) \xi^{n}=\sum_{k=0}^{\infty} \sum_{\rho} \boldsymbol{\rho} \sum_{\mathbf{r}}{ }^{\prime} \Pi^{k}\left(\boldsymbol{\rho}, \mathbf{r} \mid \mathbf{0}, \mathbf{r}_{\mathbf{0}}\right)
$$

and

$$
S\left(\mathbf{r}_{\mathbf{0}} ; \xi\right) \equiv \sum_{n=0}^{\infty}\left\langle\boldsymbol{\rho}^{2}\right\rangle_{n}\left(\mathbf{r}_{\mathbf{0}}\right) \xi^{n}=\sum_{k=0}^{\infty} \sum_{\rho} \boldsymbol{\rho}^{2} \sum_{\mathbf{r}}^{\prime} \Pi^{k}\left(\boldsymbol{\rho}, \mathbf{r} \mid \mathbf{0}, \mathbf{r}_{\mathbf{0}}\right)
$$

By peforming the sums over $\mathbf{r}$ and $\boldsymbol{\rho}$ we arrive at the final expressions for $\mathbf{A}\left(\mathbf{r}_{\mathbf{0}} ; \xi\right)$ and $S\left(\mathbf{r}_{\mathbf{0}} ; \xi\right)$ (see Appendix D):

$$
\begin{gathered}
\mathbf{A}\left(\mathbf{r}_{\mathbf{0}} ; \xi\right)=\frac{1}{1-\xi} \frac{1}{1-q} \mathbf{M}\left(\mathbf{r}_{\mathbf{0}} ; \xi\right) \\
S\left(\mathbf{r}_{\mathbf{0}} ; \xi\right)=\frac{1}{1-\xi} \frac{1}{1-u / t} \frac{1+q}{1-q} \frac{P\left(\mathbf{0} \mid \mathbf{r}_{\mathbf{0}} ; \xi\right)}{t}
\end{gathered}
$$

where

$$
\mathbf{M}\left(\mathbf{r}_{\mathbf{0}} ; \xi\right)=p \xi \sum_{\nu}^{(\mathbf{0})} \boldsymbol{\nu} P\left(\boldsymbol{\nu} \mid \mathbf{r}_{\mathbf{0}} ; \xi\right)
$$

and

$$
q=p \xi(h-t) .
$$

Note, for $\mathbf{r}_{\mathbf{0}}=\boldsymbol{\alpha}$ in (121) we have:

$$
\mathbf{M}(\boldsymbol{\alpha} ; \xi)=-q \boldsymbol{\alpha} .
$$


The corresponding moments will then be expressed by:

$$
\langle\boldsymbol{\rho}\rangle_{n}\left(\mathbf{r}_{\mathbf{0}}\right)=\frac{1}{2 \pi \mathrm{i}} \oint_{\Gamma} \frac{d \xi}{\xi^{n+1}} \frac{1}{1-\xi} \frac{\mathbf{M}\left(\mathbf{r}_{\mathbf{0}} ; \xi\right)}{1-q}
$$

and

$$
\left\langle\boldsymbol{\rho}^{2}\right\rangle_{n}\left(\mathbf{r}_{\mathbf{0}}\right)=\frac{1}{2 \pi \mathrm{i}} \oint_{\Gamma} \frac{d \xi}{\xi^{n+1}} \frac{1}{1-\xi} \frac{1}{1-u / t} \frac{1+q}{1-q} \frac{P\left(\mathbf{0} \mid \mathbf{r}_{\mathbf{0}} ; \xi\right)}{t} .
$$

There is a simple expression 18 for $\mathbf{M}\left(\mathbf{r}_{\mathbf{0}} ; \xi\right)$ showing that it is in fact a vector pointing along $\mathbf{r}_{\mathbf{0}}$ :

$$
\mathbf{M}\left(\mathbf{r}_{\mathbf{0}} ; \xi\right)=\mathbf{r}_{\mathbf{0}} \frac{1}{\xi} \int_{0}^{\xi} P\left(\mathbf{0} \mid \mathbf{r}_{\mathbf{0}} ; \zeta\right) d \zeta
$$

This means, that the average of the tagged walk is always shifted towards the initial position of the vacancy.

In the following we extract the $n \rightarrow \infty$ behaviour for the different cases.

$\underline{d=1}$ Without loss of generality, assume $r_{0} \geq 1$. Then, from Appendix B:

$$
\mathbf{M}\left(\mathbf{r}_{\mathbf{0}} ; \xi\right)=\left(\frac{1-\sqrt{1-\xi^{2}}}{\xi}\right)^{r_{0}} \mathbf{e}_{1}, \text { and } q=-\frac{1-\sqrt{1-\xi^{2}}}{\xi} .
$$

Therefore, for the average displacement, we find:

$$
\langle\boldsymbol{\rho}\rangle_{n}\left(\mathbf{r}_{\mathbf{0}}\right)=\frac{\mathbf{e}_{1}}{2 \pi \mathrm{i}} \oint_{\Gamma} \frac{d \xi}{\xi^{n}} \frac{1}{1-\xi} \frac{1}{1+\xi+\sqrt{1-\xi^{2}}}\left(\frac{1}{1+\sqrt{1-\xi^{2}}}\right)^{r_{0}-1}
$$

Using (19), we find immediately $\langle\rho\rangle_{\infty}\left(r_{0}\right)=1 / 2$, which is certainly correct because half of the time the tagged particle is at the origin, and half of the time is on site 1. The convergence to $1 / 2$, i.e., the $n$-behaviour in leading order is given by:

$$
\langle\rho\rangle_{n}\left(r_{0}\right) \sim \frac{1}{2}\left(e^{-\sqrt{2} \frac{r_{0}-1}{\sqrt{n}}}\right) \mathbf{e}_{1} .
$$

Observe that, as pointed out below Eq. (126) on average, the tagged particle suffers a finite displacement toward the initial position of the vacancy. One can consider this as an effective attraction between the tagged particle and the random walker. This is property valid in any dimension.

The second order moment turns out to be: $\left\langle\rho^{2}\right\rangle_{n}\left(r_{0}\right)=\langle\rho\rangle_{n}\left(r_{0}\right)$. Thus, the spread is $\mathcal{D}_{\infty}\left(r_{0}\right)=1 / 2$, as expected.

$\underline{d=2}$ To obtain explicit expressions for the general case is non-trivial. For example, if the tagged particle and the vacancy are initially very far apart, we expect that the particle will experience the first hit to come from all directions with equal probability. Then its average displacement will vanish. So, we choose to present only the case where the vacancy-tracer is initially a nearest neighbour pair, i.e., $\mathbf{r}_{\mathbf{0}}=\boldsymbol{\alpha}$, where $\boldsymbol{\alpha}$ is a nearest neighbour of the origin. 
The average displacement is then given by:

$$
\langle\boldsymbol{\rho}\rangle_{n}(\boldsymbol{\alpha})=\frac{1}{2 \pi \mathrm{i}} \oint_{\Gamma} \frac{d \xi}{\xi^{n+1}} \frac{1}{1-\xi} \frac{(-q)}{1-q} \boldsymbol{\alpha},
$$

and in the limit $n \rightarrow \infty$ (see Appendix B for the expression of $q$ ):

$$
\langle\boldsymbol{\rho}\rangle_{\infty}(\boldsymbol{\alpha})=\frac{1}{2} \frac{\pi-2}{\pi-1} \boldsymbol{\alpha}=0.266529 \boldsymbol{\alpha} .
$$

Applying The Discrete Tauberian Theorem, the second moment is found:

$$
\left\langle\boldsymbol{\rho}^{2}\right\rangle_{n}(\boldsymbol{\alpha}) \sim \frac{1}{\pi(\pi-1)} \ln n \frac{1}{1-\frac{\ln n}{n \pi}}, \quad n \gg 1 .
$$

this leads to the spread:

$$
\mathcal{D}_{n}(\boldsymbol{\alpha}) \sim\left[\frac{1}{\pi(\pi-1)} \ln n-\frac{1}{4}\left(\frac{\pi-2}{\pi-1}\right)^{2}\right]^{1 / 2} \sim \frac{1}{\sqrt{\pi(\pi-1)}} \sqrt{\ln n}, \quad n \gg 1 .
$$

i.e. it diverges as $\sqrt{\ln n}$. This is exactly the result we anticipated from the scaling given by (113).

$d \geq 3$ Since there is no divergence in this case, the first and second moments are finite even for $n=\infty$ :

$$
\langle\boldsymbol{\rho}\rangle_{\infty}\left(\mathbf{r}_{\mathbf{0}}\right)=\frac{M\left(\mathbf{r}_{\mathbf{0}} ; 1\right)}{1-q(1)}, \text { and }\left\langle\boldsymbol{\rho}^{2}\right\rangle_{\infty}\left(\mathbf{r}_{\mathbf{0}}\right)=\frac{1+q(1)}{1-q(1)} P\left(\mathbf{0} \mid \mathbf{r}_{\mathbf{0}} ; 1\right) .
$$

The spread,

$$
\mathcal{D}_{\infty}\left(\mathbf{r}_{\mathbf{0}}\right) \sim\left\{\frac{1+q(1)}{1-q(1)} P\left(\mathbf{0} \mid \mathbf{r}_{\mathbf{0}} ; 1\right)-\left[\frac{M\left(\mathbf{r}_{\mathbf{0}} ; 1\right)}{1-q(1)}\right]^{2}\right\}^{1 / 2}
$$

is also just a finite number.

If the vacancy again started from a nearest neighbour $\boldsymbol{\alpha}$ of the tagged particle, then, we have, via (123):

$$
\langle\boldsymbol{\rho}\rangle_{\infty}(\boldsymbol{\alpha})=-\frac{q(1)}{1-q(1)} \boldsymbol{\alpha}, \text { and } \mathcal{D}_{\infty}(\boldsymbol{\alpha})=\frac{1}{1-q(1)} \sqrt{\left[1-q(1)^{2}\right] t(1)-1}
$$

In particular, in three dimensions $t(1)=1.516386$ and $q(1)=-0.209$.. (see Appendix B), so that

$$
\langle\boldsymbol{\rho}\rangle_{\infty}(\boldsymbol{\alpha})=0.1728 . . \boldsymbol{\alpha}, \text { and } \mathcal{D}_{\infty}(\boldsymbol{\alpha})=0.5549 \ldots .
$$

\section{Conclusions and summary}

We investigated the walk performed by the tagged particle in the presence of a single vacancy in a d-dimensional, infinite square lattice. The only "active" object 
is the vacancy, while the tagged particle performs a "passive" walk, being shifted only when it exchanged positions with the Brownian vacancy. We constructed the complete probability distribution, which is quite complex, incorporating three spatial variables $\left(\mathbf{r}_{\mathbf{0}}, \boldsymbol{\rho}\right.$ and $\mathbf{r}$ ) and two temporal ones ( $n$ and $k$ ). However, physically relevant informations can be extracted from different projections of the full distribution. An example is $\bar{\pi}_{n}\left(\boldsymbol{\rho} \mid \mathbf{r}_{\mathbf{0}}\right)$, the probability distribution for the tagged particle to be at site $\boldsymbol{\rho}$ on the $n$-th step of the vacancy. Another is $\hat{\pi}_{n}^{k}\left(\mathbf{r}_{\mathbf{0}}\right)$, the probability that it has made $k$ steps during this time. Closed form expressions for the corresponding generating functions, in terms of the generating function of the Brownian walker alone, were obtained. Exploiting the powerful Discrete Tauberian Theorem, we find the limiting behaviours of the distributions themselves, as $n \rightarrow \infty$. We showed that the Brownian vacancy driven walk is reccurent in two dimensions only. Other interesting results associated with a tagged particle in $d=2$ are

(i) the number of steps it takes scales as $\ln n($ as $n \rightarrow \infty)$

(ii) the limiting displacement- distribution is not a Gaussian but rather the modified Bessel function $K_{0}$

(iii) its standard deviation scales as $\sqrt{\ln n}$, in contrast to the usual $\sqrt{n}$ scaling Brownian walks

By computing the first moment for the displacement-distribution of the tagged particle, we find that there is an effective attraction towards the vacancy which shifts the average position of the tagged particle from its initial point. In $d=1$, 2 , and 3, this shift is, respectively, 0.5, 0.266529.., 0.1728.. in units of the lattice spacing. For higher dimensions this is given by the first expression in (134). We have seen, that an immediate consequence of this attraction is the tagged particle being visited more frequently than its original site. However the difference vanishes as $n \rightarrow \infty$, for $d \geq 2$.

In $d \geq 3$, the standard deviation of the displacement-distribution does not diverge as $n \rightarrow \infty$. Instead, it converges to a small number, e.g., 0.5549 in $d=3$.

\section{Acknowledgements}

Illuminating discussions with R. K. P. Zia, B. Schmittmann, G. Korniss, C. Laberge, and S. Sandow are gratefully acknowledged. This research is supported in part by the US National Science Foundation through the Division of Materials Research and the Hungarian Science Foundation under grant numbers OTKA F17166 and T17493.

\section{Appendix A}

\section{Normalization}

When proving (29) it is better to switch to generating functions. (29) is equiv- 
alent to:

$$
\sum_{k=0}^{\infty} \sum_{\rho} \sum_{\mathbf{r}}^{\prime} \delta_{\mathbf{R}, \rho+\mathbf{r}} \Pi^{k}\left(\boldsymbol{\rho}, \mathbf{r} \mid \mathbf{0}, \mathbf{r}_{\mathbf{0}} ; \xi\right)=P\left(\mathbf{R} \mid \mathbf{r}_{\mathbf{0}} ; \xi\right)
$$

Separate the $k=0$ term in A.1):

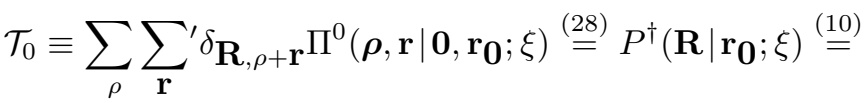

$$
\begin{aligned}
& P\left(\mathbf{R} \mid \mathbf{r}_{\mathbf{0}} ; \xi\right)-\frac{1}{t} P(\mathbf{R} \mid \mathbf{0} ; \xi) P\left(\mathbf{0} \mid \mathbf{r}_{\mathbf{0}} ; \xi\right) .
\end{aligned}
$$

As for the rest $(\boldsymbol{\rho}=\mathbf{R}-\mathbf{r})$ :

$$
\begin{aligned}
\mathcal{T} \equiv & \sum_{k=1}^{\infty} \sum_{\rho} \sum_{\mathbf{r}}{ }^{\prime} \delta \mathbf{R}, \rho+\mathbf{r} \Pi^{k}\left(\boldsymbol{\rho}, \mathbf{r} \mid \mathbf{0}, \mathbf{r}_{\mathbf{0}} ; \xi\right)=\int_{-\pi}^{\pi} \frac{d^{d} l}{(2 \pi)^{d}} e^{\mathrm{i} \mathbf{l} \mathbf{R}} \sum_{k=1}^{\infty}(p \xi)^{k} \sum_{\nu_{1}}^{(\mathbf{0})} \ldots \sum_{\nu_{k}}^{(\mathbf{0})} \\
& {\left[\sum_{\mathbf{r}}^{\prime} e^{-\mathrm{i} \mathbf{l} \mathbf{r}^{\dagger}} P^{\dagger}\left(\mathbf{r} \mid-\boldsymbol{\nu}_{k} ; \xi\right)\right] e^{-\mathrm{i} \mathbf{l} \nu_{k}} P^{\dagger}\left(\boldsymbol{\nu}_{k} \mid-\boldsymbol{\nu}_{k-1} ; \xi\right) \ldots e^{-\mathrm{i} \mathbf{l}_{1}} P^{\dagger}\left(\boldsymbol{\nu}_{1} \mid \mathbf{r}_{\mathbf{0}} ; \xi\right) . }
\end{aligned}
$$

Using Eq. (21) with $\boldsymbol{\alpha}=-\boldsymbol{\nu}_{k}$, the term in square bracket is computed, and:

$$
\begin{aligned}
\mathcal{T}= & \int_{-\pi}^{\pi} \frac{d^{d} l}{(2 \pi)^{d}} \frac{e^{\mathrm{i} \mathbf{l} \mathbf{R}}}{1-\xi \omega(\mathbf{l})} \sum_{k=1}^{\infty}(p \xi)^{k} \sum_{\nu_{1}}^{(\mathbf{0})} \sum_{\nu_{2}}^{(\mathbf{0})} \ldots \sum_{\nu_{k}}^{(\mathbf{0})}\left[1-\frac{u}{t} e^{-\mathrm{i} \mathbf{l} \nu_{k}}\right] \\
& P^{\dagger}\left(\boldsymbol{\nu}_{k} \mid-\boldsymbol{\nu}_{k-1} ; \xi\right) \ldots e^{-\mathrm{i} \mathbf{l}_{2}} P^{\dagger}\left(\boldsymbol{\nu}_{2} \mid-\boldsymbol{\nu}_{1} ; \xi\right) e^{-\mathrm{i} \mathbf{l}_{\nu_{1}}} P^{\dagger}\left(\boldsymbol{\nu}_{1} \mid \mathbf{r}_{\mathbf{0}} ; \xi\right) .
\end{aligned}
$$

According to the square bracket in A.4 , $\mathcal{T}$ splits in two terms $\left(\mathcal{T}=\theta_{1}+\theta_{2}\right)$. Using notation (46), the second term becomes:

$$
\theta_{2} \equiv-\frac{u}{t} \int_{-\pi}^{\pi} \frac{d^{d} l}{(2 \pi)^{d}} \frac{e^{\mathrm{i} \mathbf{l} \mathbf{R}}}{1-\xi \omega(\mathbf{l})} \Psi_{\mathbf{l}}(\mathbf{0})
$$

while the first:

$$
\begin{aligned}
& \theta_{1} \equiv \int_{-\pi}^{\pi} \frac{d^{d} l}{(2 \pi)^{d}} \frac{e^{\mathrm{i} \mathbf{l} \mathbf{R}}}{1-\xi \omega(\mathbf{l})} \sum_{k=1}^{\infty} p^{k} \xi^{k} \sum_{\nu_{1}}^{(\mathbf{0})} \sum_{\nu_{2}}^{(\mathbf{0})} \ldots\left[\sum_{\nu_{k}}^{(\mathbf{0})} P^{\dagger}\left(\boldsymbol{\nu}_{k} \mid-\boldsymbol{\nu}_{k-1} ; \xi\right)\right]
\end{aligned}
$$

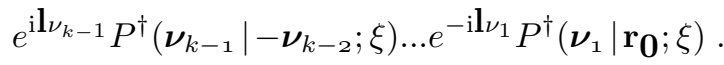

We again need to separate the $k=1$ term in $\theta_{1}$ :

$$
\theta_{1}=\int_{-\pi}^{\pi} \frac{d^{d} l}{(2 \pi)^{d}} \frac{e^{\mathrm{i} \mathbf{l} \mathbf{R}}}{1-\xi \omega(\mathbf{l})} p \xi\left\{\sum_{\nu_{1}}^{(\mathbf{0})} P^{\dagger}\left(\boldsymbol{\nu}_{1} \mid \mathbf{r}_{\mathbf{0}} ; \xi\right)\right\}+(k \geq 2 \text { terms }) .
$$


The term in square brackets in (A.6) is computed via (25), while the term in curly brackets in A.7 is expressed by (24). Combining these, we finally get for the first term:

$$
\begin{aligned}
& \theta_{1}=\frac{1}{t} P(\mathbf{R} \mid \mathbf{0} ; \xi) P\left(\mathbf{0} \mid \mathbf{r}_{\mathbf{0}} ; \xi\right)+\frac{u}{t} \int_{-\pi}^{\pi} \frac{d^{d} l}{(2 \pi)^{d}} \frac{e^{\mathrm{i} \mathbf{l}}}{1-\xi \omega(\mathbf{l})} \sum_{k=2}^{\infty}(p \xi)^{k-1} \sum_{\nu_{1}}^{(\mathbf{0})} \sum_{\nu_{2}}^{(\mathbf{0})} \ldots \sum_{\nu_{k-1}}^{(\mathbf{0})}
\end{aligned}
$$

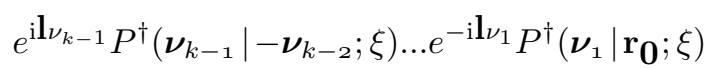

$$
\begin{aligned}
& =\frac{1}{t} P(\mathbf{R} \mid \mathbf{0} ; \xi) P\left(\mathbf{0} \mid \mathbf{r}_{\mathbf{0}} ; \xi\right)+\frac{u}{t} \int_{-\pi}^{\pi} \frac{d^{d} l}{(2 \pi)^{d}} \frac{e^{\mathrm{i} \mathbf{l} \mathbf{R}}}{1-\xi \omega(\mathbf{l})} \Psi_{\mathbf{l}}(\mathbf{0}) .
\end{aligned}
$$

Adding the first $\left(\theta_{1}\right)$ and the second term $\left(\theta_{2}\right)$, is obtained:

$$
\mathcal{T}=\frac{1}{t} P(\mathbf{R} \mid \mathbf{0} ; \xi) P\left(\mathbf{0} \mid \mathbf{r}_{\mathbf{0}} ; \xi\right) .
$$

(the terms containing the $\Psi$-s cancel). Add (A.9) to (A.2) and after cancellation, (A.1) is gained. Therefore normalization (A.1) holds.

\section{Appendix B}

\section{Generating functions}

For dimension one, the explicit expression for the $P(0 \mid R ; \xi)$ generating function is well known $\mathrm{G}$ :

$$
P(0 \mid R ; \xi)=t\left(\frac{1-\sqrt{1-\xi^{2}}}{\xi}\right)^{|R|}
$$

with:

$$
t=P(0 \mid 0 ; \xi)=\left(1-\xi^{2}\right)^{-\frac{1}{2}}
$$

Thus, according to (7) (or (17) ), and (8):

$$
u=t \frac{1-\sqrt{1-\xi^{2}}}{\xi}, \quad h=\frac{u^{2}}{t} .
$$

In two dimensions 615 :

$$
t=P(\mathbf{0} \mid \mathbf{0} ; \xi)=\frac{2}{\pi} \mathbf{K}(\xi)
$$

where $\mathbf{K}(\xi)$ is the complete elliptic integral of first kind. Then, according to (17):

$$
u=\frac{1}{\xi}\left[\frac{2}{\pi} \mathbf{K}(\xi)-1\right] .
$$

Quoting Ref. 15, the probabilities $P(\mathbf{0} \mid \mathbf{R} ; \xi)$ with $\mathbf{R}=\mathbf{R}(m, m), m \in Z\left(\mathbf{R}\left(m_{1}, m_{2}\right)\right.$ is a lattice vector with components $m_{1}$ and $\left.m_{2}\right)$ can be expressed in terms of Legendre functions of second kind:

$$
P(\mathbf{0} \mid \mathbf{R}(m, m) ; \xi)=\frac{2}{\pi \xi} Q_{m-1 / 2}\left(\frac{1-\xi^{2} / 2}{\xi^{2} / 2}\right) .
$$


Note, that for the Pólya walk $P\left(\mathbf{0} \mid \mathbf{R}\left(m_{1}, m_{2}\right) ; \xi\right)=P\left(\mathbf{0} \mid \mathbf{R}\left(m_{1},-m_{2}\right) ; \xi\right)=P(\mathbf{0} \mid$ $\left.\mathbf{R}\left(-m_{1}, m_{2}\right) ; \xi\right)=P\left(\mathbf{0} \mid \mathbf{R}\left(-m_{1},-m_{2}\right) ; \xi\right)$ for any $m_{1}, m_{2} \in Z$.

For $m=0$ we regain (B.4). For $m=1$ :

$$
b \equiv P(\mathbf{0} \mid R(1,1) ; \xi)=\frac{4}{\pi \xi^{2}}\left[\left(1-\frac{\xi^{2}}{2}\right) \mathbf{K}(\xi)-\mathbf{E}(\xi)\right],
$$

with $\mathbf{E}(\xi)$ as the complete elliptic integral of second kind.

From (18) follows:

$$
h=\frac{2}{\pi} \mathbf{K}(\xi)+\frac{4}{\xi^{2}}\left[\frac{2}{\pi} \mathbf{E}(\xi)-1\right] .
$$

Therefore, from (122):

$$
q=\frac{1}{\xi}\left[\frac{2}{\pi} \mathbf{E}(\xi)-1\right] .
$$

When $\xi \rightarrow 1^{-}, t, u, b$ and $h$ all diverge, since $\mathbf{K}(\xi)$ has a logarithmic singularity in $\xi=1$. Accordingly, we have the following asimptotic behaviour 16 :

$$
t(\xi) \sim \frac{1}{\pi} \ln \frac{8}{1-\xi}, \quad \text { as } \quad \xi \rightarrow 1^{-} .
$$

Note that for $\xi=1, q(1)=-(1-2 / \pi)=-0.36338$.

In dimensions three the value for $t(1)$ is given analitically 6 as $t(1)=\frac{\sqrt{6}}{32 \pi^{3}} \Gamma\left(\frac{1}{24}\right)$ $\Gamma\left(\frac{5}{24}\right) \Gamma\left(\frac{7}{24}\right) \Gamma\left(\frac{11}{24}\right)=1.516386$. We numerically estimated the value of $b(1)$ as $b(1)=$ 0.331 . Thus, from equation (18) $t(1)+4 b(1)+h(1)=6 u(1)$, and one finds $h(1)=$ 0.2579 . Therefore $q(1)=-0.209$.

\section{Appendix C}

\section{Diagonalization of $\mathrm{P}$}

$\mathbf{P}$ is easily diagonalised. It has three different eigenvalues (for $d=1$ only two, $\Lambda_{1}$ and $\left.\Lambda_{3}\right)$ :

$$
\begin{aligned}
\lambda_{1}=\Lambda_{1} & =h+2(d-1) b+t, \\
\lambda_{2}=\lambda_{3}=\ldots=\lambda_{d}=\Lambda_{2} & =h-2 b+t, \\
\lambda_{d+1}=\lambda_{d+2}=\ldots=\lambda_{2 d}=\Lambda_{3} & =h-t .
\end{aligned}
$$

The corresponding system of orthonormal eigenvectors is:

$$
\left[\phi_{1}\right]^{T}=\frac{1}{\sqrt{2 d}}\left[\begin{array}{lll|lll}
1 & \cdots & 1 & 1 & \cdots & 1
\end{array}\right],
$$

as the single eigenvector corresponding to $\Lambda_{1}$,

$$
\left[\phi_{j}\right]^{T}=\frac{1}{\sqrt{2 j(j-1)}}\left[\begin{array}{l|l}
-10 . .0(j-1)-1 . .-1 & -10 . .0(j-1)-1 . .-1 \\
j \in\{2,3, \ldots, d\}, \quad(\mathrm{C} .5)
\end{array}\right.
$$


as the $d-1$ eigenvectors corresponding to $\Lambda_{2}$ (the small vertical line is between elements $d$ and $d+1)$. The elements -1 in $\left[\phi_{j}\right]^{T}$ are in the columns $1, d-j+3$, $d-j+4, \ldots, d-1, d$ and $d+1,2 d-j+3,2 d-j+4, \ldots, 2 d-1,2 d$, respectively, while the elements $j-1$ are at the columns $d-j+2$ and $2 d-j+2$, respectively. And, finally,

$$
\begin{aligned}
& {\left[\phi_{i}\right]^{T}=\frac{1}{\sqrt{2}}\left[\begin{array}{lllll|lllll}
0 & \cdots & -1 & \cdots & 0 & 0 & \cdots & 1 & \cdots & 0
\end{array}\right],} \\
& i \in\{d+1, d+2, \ldots, 2 d\}
\end{aligned}
$$

are the $d$ eigenvectors corresponding $\Lambda_{3}$, with nonzero elements at columns $2 d+1-i$ and $3 d+1-i$. It is certainly valid:

$$
\left[\phi_{i}\right]^{T}\left[\phi_{j}\right]=\delta_{i, j}, \quad i, j \in\{1,2,3, \ldots, 2 d\} .
$$

$\underline{d=1}$. The eigenvalues with the corresponding eigenvectors are:

$$
\begin{gathered}
\lambda_{1}=h+t=\frac{2}{\xi} u, \quad\left[\phi_{1}\right]=\frac{1}{\sqrt{2}}\left[\begin{array}{c}
1 \\
1
\end{array}\right], \quad \text { and } \\
\lambda_{2}=h-t=-\frac{2 u}{\xi t}, \quad\left[\phi_{2}\right]=\frac{1}{\sqrt{2}}\left[\begin{array}{c}
-1 \\
1
\end{array}\right]
\end{gathered}
$$

with $t$ and $u$ given by Eqs. (B.2) and (B.3). While $\lambda_{1}$ diverges as $\xi \rightarrow 1^{-}, \lambda_{2}$ is convergent $(u / t \rightarrow 1)$.

$\underline{d=2}$. In this case the eigenvalues are given by:

$$
\begin{aligned}
& \lambda_{1}=h+2 b+t=\frac{4}{\xi} u=\frac{4}{\xi^{2}}\left[\frac{2}{\pi} \mathbf{K}(\xi)-1\right], \\
& \lambda_{2}=h-2 b+t=\frac{4}{\xi^{2}}\left[\frac{4}{\pi} \mathbf{E}(\xi)-1-\left(1-\xi^{2}\right) \frac{2}{\pi} \mathbf{K}(\xi)\right] \equiv \frac{4}{\xi} V, \\
& \lambda_{3}=\lambda_{4}=h-t=\frac{4}{\xi^{2}}\left[\frac{2}{\pi} \mathbf{E}(\xi)-1\right] \equiv \frac{4}{\xi} W,
\end{aligned}
$$

and the corresponding eigenvectors by:

$$
\left[\phi_{1}\right]=\frac{1}{2}\left[\begin{array}{l}
1 \\
1 \\
1 \\
1
\end{array}\right],\left[\phi_{2}\right]=\frac{1}{2}\left[\begin{array}{r}
-1 \\
1 \\
-1 \\
1
\end{array}\right],\left[\phi_{3}\right]=\frac{1}{\sqrt{2}}\left[\begin{array}{r}
0 \\
-1 \\
0 \\
1
\end{array}\right],\left[\phi_{4}\right]=\frac{1}{\sqrt{2}}\left[\begin{array}{r}
-1 \\
0 \\
1 \\
0
\end{array}\right] .
$$

\section{Appendix D}

\section{First and second moments}


Using the expressions (27) and (28) for the generating functions, and performing the sums over $\mathbf{r}$ and $\boldsymbol{\rho}$, we have:

$$
\mathbf{A}\left(\mathbf{r}_{\mathbf{0}} ; \xi\right)=\frac{1-u / t}{1-\xi} \sum_{k=1}^{\infty}(p \xi)^{k} \sum_{\nu_{1}}^{(\mathbf{0})} \ldots \sum_{\nu_{k}}^{(\mathbf{0})}\left(\boldsymbol{\nu}_{1}+\ldots+\boldsymbol{\nu}_{k}\right) P^{\dagger}\left(\boldsymbol{\nu}_{k} \mid-\boldsymbol{\nu}_{k-1} ; \xi\right) \ldots P^{\dagger}\left(\boldsymbol{\nu}_{1} \mid \mathbf{r}_{\mathbf{0}} ; \xi\right) .
$$

Using (10) and the notation (121), the $k=1$ term is:

$$
\frac{1-u / t}{1-\xi} p \xi \sum_{\nu_{1}}^{(\mathbf{0})} \boldsymbol{\nu}_{1} P^{\dagger}\left(\boldsymbol{\nu}_{1} \mid \mathbf{r}_{\mathbf{0}} ; \xi\right)=\frac{1-u / t}{1-\xi} \mathbf{M}\left(\mathbf{r}_{\mathbf{0}} ; \xi\right) \text {. }
$$

Defining:

$$
\mathbf{A}_{\mathbf{1}}\left(\mathbf{r}_{\mathbf{0}} ; \xi\right) \equiv \sum_{k=2}^{\infty}(p \xi)^{k} \sum_{\nu_{1}}^{(\mathbf{0})} \ldots \sum_{\nu_{k}}^{(\mathbf{0})}\left(\boldsymbol{\nu}_{1}+\ldots+\boldsymbol{\nu}_{k-1}\right) P^{\dagger}\left(\boldsymbol{\nu}_{k} \mid-\boldsymbol{\nu}_{k-1} ; \xi\right) \ldots P^{\dagger}\left(\boldsymbol{\nu}_{1} \mid \mathbf{r}_{\mathbf{0}} ; \xi\right)
$$

and

$$
\mathbf{A}_{\mathbf{2}}\left(\mathbf{r}_{\mathbf{0}} ; \xi\right) \equiv \sum_{k=2}^{\infty}(p \xi)^{k} \sum_{\nu_{1}}^{(\mathbf{0})} \ldots \sum_{\nu_{k}}^{(\mathbf{0})} \boldsymbol{\nu}_{k} P^{\dagger}\left(\boldsymbol{\nu}_{k} \mid-\boldsymbol{\nu}_{k-1} ; \xi\right) \ldots P^{\dagger}\left(\boldsymbol{\nu}_{1} \mid \mathbf{r}_{\mathbf{0}} ; \xi\right),
$$

(D.1) is rewritten as:

$$
\mathbf{A}\left(\mathbf{r}_{\mathbf{0}} ; \xi\right)=\frac{1-u / t}{1-\xi}\left[\mathbf{M}\left(\mathbf{r}_{\mathbf{0}} ; \xi\right)+\mathbf{A}_{\mathbf{1}}\left(\mathbf{r}_{\mathbf{0}} ; \xi\right)+\mathbf{A}_{\mathbf{2}}\left(\mathbf{r}_{\mathbf{0}} ; \xi\right)\right] .
$$

It is easy to see that:

$$
\mathbf{A}_{\mathbf{1}}\left(\mathbf{r}_{\mathbf{0}} ; \xi\right)=\frac{u}{t} \frac{1-\xi}{1-u / t} \mathbf{A}\left(\mathbf{r}_{\mathbf{0}} ; \xi\right) .
$$

With the aid of formulas (25) and (121) for $\mathbf{A}_{\mathbf{2}}\left(\mathbf{r}_{\mathbf{0}} ; \xi\right)$ we obtain:

$$
\mathbf{A}_{\mathbf{2}}\left(\mathbf{r}_{\mathbf{0}} ; \xi\right)=\frac{q}{1-q} \mathbf{M}\left(\mathbf{r}_{\mathbf{0}} ; \xi\right) \text {. }
$$

Inserting (D.6) and (D.7) into (D.5), (119) is gained.

Proceeding similarly as for the first moment, the generating function for the second moment becomes:

$$
S\left(\mathbf{r}_{\mathbf{0}} ; \xi\right)=\frac{1-u / t}{1-\xi} \sum_{k=1}^{\infty}(p \xi)^{k} \sum_{\nu_{1}}^{(\mathbf{0})} \ldots \sum_{\nu_{k}}^{(\mathbf{0})}\left(\boldsymbol{\nu}_{1}+\ldots+\boldsymbol{\nu}_{k}\right)^{2} P^{\dagger}\left(\boldsymbol{\nu}_{k} \mid-\boldsymbol{\nu}_{k-1} ; \xi\right) \ldots P^{\dagger}\left(\boldsymbol{\nu}_{1} \mid \mathbf{r}_{\mathbf{0}} ; \xi\right) .
$$

Separating the term $k=1$ in $(\overline{D .8})$ and using the fact that $\left(\boldsymbol{\nu}_{1}+\ldots+\boldsymbol{\nu}_{k}\right)^{2}=$ $\left(\boldsymbol{\nu}_{1}+\ldots+\boldsymbol{\nu}_{k-1}\right)^{2}+2\left(\boldsymbol{\nu}_{1}+\ldots+\boldsymbol{\nu}_{k-1}\right)+1\left(\boldsymbol{\nu}_{i}{ }^{2}=1, \forall i\right), S\left(\mathbf{r}_{\mathbf{0}} ; \xi\right)$ is rewritten as:

$$
S\left(\mathbf{r}_{\mathbf{0}} ; \xi\right)=\frac{1-u / t}{1-\xi}\left[\frac{P\left(\mathbf{0} \mid \mathbf{r}_{\mathbf{0}} ; \xi\right)}{t}+S_{1}\left(\mathbf{r}_{\mathbf{0}} ; \xi\right)+2 S_{2}\left(\mathbf{r}_{\mathbf{0}} ; \xi\right)+S_{3}\left(\mathbf{r}_{\mathbf{0}} ; \xi\right)\right],
$$


where

$$
\begin{gathered}
S_{1}\left(\mathbf{r}_{\mathbf{0}} ; \xi\right)=\sum_{k=2}^{\infty}(p \xi)^{k} \sum_{\nu_{1}}^{(\mathbf{0})} \ldots \sum_{\nu_{k}}^{(\mathbf{0})}\left(\boldsymbol{\nu}_{1}+\ldots+\boldsymbol{\nu}_{k-1}\right)^{2} P^{\dagger}\left(\boldsymbol{\nu}_{k} \mid-\boldsymbol{\nu}_{k-1} ; \xi\right) \ldots P^{\dagger}\left(\boldsymbol{\nu}_{1} \mid \mathbf{r}_{\mathbf{0}} ; \xi\right), \\
S_{2}\left(\mathbf{r}_{\mathbf{0}} ; \xi\right)=\sum_{k=2}^{\infty}(p \xi)^{k} \sum_{\nu_{1}}^{(\mathbf{0})} \ldots \sum_{\nu_{k}}^{(\mathbf{0})}\left(\boldsymbol{\nu}_{1}+\ldots+\boldsymbol{\nu}_{k-1}\right) \boldsymbol{\nu}_{k} P^{\dagger}\left(\boldsymbol{\nu}_{k} \mid-\boldsymbol{\nu}_{k-1} ; \xi\right) \ldots P^{\dagger}\left(\boldsymbol{\nu}_{1} \mid \mathbf{r}_{\mathbf{0}} ; \xi\right), \\
S_{3}\left(\mathbf{r}_{\mathbf{0}} ; \xi\right)=\sum_{k=2}^{\infty}(p \xi)^{k} \sum_{\nu_{1}}^{(\mathbf{0})} \ldots \sum_{\nu_{k}}^{(\mathbf{0})} P^{\dagger}\left(\boldsymbol{\nu}_{k} \mid-\boldsymbol{\nu}_{k-1} ; \xi\right) \ldots P^{\dagger}\left(\boldsymbol{\nu}_{1} \mid \mathbf{r}_{\mathbf{0}} ; \xi\right),
\end{gathered}
$$

In virtue of (24) and (25) is not hard to see that:

$$
S_{1}\left(\mathbf{r}_{\mathbf{0}} ; \xi\right)=\frac{u}{t} \frac{1-\xi}{1-u / t} S\left(\mathbf{r}_{\mathbf{0}} ; \xi\right), \quad S_{2}\left(\mathbf{r}_{\mathbf{0}} ; \xi\right)=\frac{q}{1-q} \frac{1}{1-u / t} \frac{P\left(\mathbf{0} \mid \mathbf{r}_{\mathbf{0}} ; \xi\right)}{t}
$$

and

$$
S_{3}\left(\mathbf{r}_{\mathbf{0}} ; \xi\right)=\frac{u}{t} \frac{1}{1-u / t} \frac{P\left(\mathbf{0} \mid \mathbf{r}_{\mathbf{0}} ; \xi\right)}{t},
$$

Inserting these into (D.9), 120 follows.

\section{References}

[1] C. A. Sholl, J. Phys. C 14, 2723 (1981)

[2] S. Alexander and P. Pincus, Phys.Rev.B 18, 2011 (1978)

[3] H.van Beijeren, K.W. Kehr, and R.Kutner, Phys.Rev.B 28, 5711 (1983)

[4] K.W. Kehr, R.Kutner, and K. Binder, Phys.Rev.B, 23, 4931, (1981)

[5] R.G. Palmer, D.L. Stein, E. Abrahams, and P.W. Anderson, Phys. Rev.Lett. 53, 958 (1984)

[6] B.D. Hughes, Random Walks and Random Environments Volume 1: Random Walks, (Oxford: Clarendon Press,1995)

[7] G. Pólya, l'Enseignement Mathématique 20, 44, (1919)

[8] G. Pólya, Mathematische Annalen 83, 149, (1921)

[9] Z. Toroczkai, Ph.D. Dissertation, Virginia Tech., 1997.

[10] W. Feller, An Introduction to Probability Theory and its Applications, Vol. 2, 2nd edition (New York: Wiley, 1970)

[11] C. Itzykson and J.- M. Drouffe, Statistical Field Theory, Vol. 1: From Brownian motion to renormalization and lattice gauge theory (Cambridge University Press, 1989)

[12] D.S. Jones, The theory of generalised functions (Cambridge University Press, 1982)

[13] G.H. Hardy, Divergent Series. (Oxford: Clarendon Press, 1949);

[14] D. V. Widder, The Laplace Transform., (Princeton University Press, 1946)

[15] E. W. Montroll, in Proceedings of Symposia on Applied Mathematics 16, 193, (1964)

[16] I. S. Gradshteyn and I. M. Ryzhik, Table of Integrals, Series, and Products, fifth edition (New York, Academic Press, 1994)

[17] M. J. A. M. Brummelhuis and H. J. Hilhorst J.Stat.Phys. 53, 249 (1988)

[18] R.K.P. Zia, unpublished.

[19] Z. Toroczkai, G. Korniss, B. Schmittmann and R.K.P. Zia, Europhys.Lett., Brownian Vacancy Mediated Disordering Dynamics submitted for publication (1997) 